\title{
ITERATION OF MEROMORPHIC FUNCTIONS
}

\author{
WALTER BERGWEILER
}

\section{Contents}

1. Introduction

2. Fatou and Julia Sets

2.1. The definition of Fatou and Julia sets

2.2. Elementary properties of Fatou and Julia sets

3. Periodic Points

3.1. Definitions

3.2. Existence of periodic points

3.3. The Julia set is perfect

3.4. Julia's approach

4. The Components of the Fatou set

4.1. The types of domains of normality

4.2. The classification of periodic components

4.3. The role of the singularities of the inverse function

4.4. The connectivity of the components of the Fatou set

4.5. Wandering domains

4.6. Classes of functions without wandering domains

4.7. Baker domains

4.8. Classes of functions without Baker domains

4.9. Completely invariant domains

5. Properties of the Julia Set

5.1. Cantor sets and real Julia sets

5.2. Points that tend to infinity

5.3. Cantor bouquets

6. Newton's Method

6.1. The unrelaxed Newton method

6.2. The relaxed Newton method

7. Miscellaneous topics

\section{References}

Received by the editors February 23, 1993 and, in revised form, May 1, 1993.

1991 Mathematics Subject Classification. Primary 30D05, 58F08; Secondary 30D30, 65H05.

Key words and phrases. Iteration, meromorphic function, entire function, set of normality, Fatou set, Julia set, periodic point, wandering domain, Baker domain, Newton's method. 


\section{INTRODUCTION}

Mathematical models for phenomena in the natural sciences often lead to iteration. An often-quoted example (compare [105]) comes from population biology. Assuming that the size of a generation of a population depends solely on the size of the previous generation and may thus be expressed as a function of it, questions concerning the further development of the population reduce to iteration of this function. More often, a phenomenon from physics or other sciences is described by a differential equation. In certain cases, for example, if there is a periodic solution, this differential equation may be studied by looking at a Poincaré return map (see, e.g., $[118, \S 1.4]$ ), and again we are led to iteration. If we solve the differential equation numerically, we are also likely to use a method based on iteration. In fact, many algorithms of numerical analysis (not only those for solving differential equations) involve iteration. One such algorithm, Newton's method of finding zeros, will be discussed in some detail in $\S 6$. Apart from that section, however, we will mainly study iteration theory in its own right without having specific applications in mind. On the other hand, it is hoped that the questions considered here may also serve as models for other situations so that their study will enhance our knowledge of dynamical systems in general.

There are two basic problems in iteration theory. The first (and classical) one is to study the iterative behavior of an individual function; the second one is to study how the behavior changes if the function is perturbed, the simplest (but already sufficiently complicated) case being a family of functions that depends on one parameter. Although the second aspect has received much attention in recent years, we shall consider here only the first one, except for a few short remarks in $\S 7$. On the other hand, a good understanding of the dynamics of an individual function is of course necessary for the study of problems involving perturbation of functions. We shall restrict ourselves to functions of one complex variable that are meromorphic in the complex plane. This includes rational and entire functions as special cases.

Although some work on iteration was already done in the last century, it is fair to say that the iteration theory of rational functions originated with the work of Fatou [71] and Julia [89], who published long memoirs on the subject between 1918 and 1920. At least Fatou's motivation was partly to study functional equations, yet another reason to consider iteration theory. At the same time, the iteration of rational functions was also investigated by Ritt [116]. Some years later in 1926 Fatou [72] extended some of the results to the case of transcendental entire functions. He did not, however, consider transcendental meromorphic functions, because [72, p. 337] here, in general, the iterates have infinitely many essential singularities. Julia did not consider the iteration of transcendental functions at all. (As already pointed out by Fatou [72, p. 358], there occurs a serious difficulty when one tries to generalize Julia's approach to the transcendental case; see the discussion in $\S 3.4$.)

In the past decade there was a renewed interest in the iteration theory of analytic functions, partially due to the beautiful computer graphics related to it (see, for example, the book by Peitgen and Richter [112]), partially due to new and powerful mathematical methods introduced into it (notably those intro- 
duced by Douady and Hubbard [62] and by Sullivan [129]). Most of the work has centered around the iteration theory of rational functions, but there is also a considerable number of papers devoted to transcendental entire functions; and in recent years work on the iteration of transcendental meromorphic functions has also begun.

There exist a number of introductions to and surveys of the iteration theory of rational functions. We mention [27, 37, 42, 53, 63, 69, 93, 100, 108, 128] among the more recent ones but also some older ones $[40,46,110]$. There are comparatively few expositions of the iteration theory of transcendental functions. We refer to [18] for the iteration of entire functions and to [69], which has a chapter on this topic.

This paper attempts to describe some of the results obtained in the iteration theory of transcendental meromorphic functions, not excluding the case of entire functions. The reader is not expected to be familiar with the iteration theory of rational functions. On the other hand, some aspects where the transcendental case is analogous to the rational case are treated rather briefly here. For example, we introduce the different types of components of the Fatou set that occur in the iteration of rational functions but omit a detailed description of these types. Instead, we concentrate on the types of components that are special to transcendental functions (Baker domains and wandering domains).

This article is mainly an exposition of known results, but it also contains some new results. For example, Theorems 5 and 16 have been known before only for entire functions or special classes of meromorphic functions. Other results like Theorem 10 or Corollaries 1 or 2 are certainly known to those who work in the field, but they do not seem to have been stated explicitly before.

As already mentioned, there are beautiful computer graphics related to the theory, and there are many places (besides [112]) where such pictures can be found for rational functions. Although Julia sets (and bifurcation diagrams) of transcendental functions can compete in their beauty and complexity very well with those of rational functions, this article is not illustrated with such pictures. The interested reader is referred to [53-56, 96].

\section{FAtou AND Julia SETS}

2.1. The definition of Fatou and Julia sets. Let $f: \mathbb{C} \rightarrow \widehat{\mathbb{C}}$ be a meromorphic function, where $\mathbb{C}$ is the complex plane and $\widehat{\mathbb{C}}=\mathbb{C} \cup\{\infty\}$. Throughout this paper, we shall always assume that $f$ is neither constant nor a linear transformation. Denote by $f^{n}$ the $n$th iterate of $f$, that is, $f^{0}(z)=z$ and $f^{n}(z)=f\left(f^{n-1}(z)\right)$ for $n \geq 1$. Then $f^{n}(z)$ is defined for all $z \in \mathbb{C}$ except for a countable set which consists of the poles of $f, f^{2}, \ldots, f^{n-1}$. If $f$ is rational, then $f$ has a meromorphic extension to $\widehat{\mathbb{C}}$; and, denoting the extension again by $f$, we see that $f^{n}$ is defined and meromorphic in $\widehat{\mathbb{C}}$. But if $f$ is transcendental-and this is the case we are mainly interested in-there is, of course, no (reasonable) way to define $f(\infty)$.

The basic objects studied in iteration theory are the Fatou set $F=F(f)$ and the Julia set $J=J(f)$ of a meromorphic function $f$. Roughly speaking, the Fatou set is the set where the iterative behavior is relatively tame in the sense that points close to each other behave similarly, while the Julia set is the set 
where chaotic phenomena take place. The formal definitions are $F=\left\{z \in \widehat{\mathbb{C}}:\left\{f^{n}: n \in \mathbb{N}\right\}\right.$ is defined and normal in some neighborhood of $\left.z\right\}$ and

$$
J=\widehat{\mathbb{C}} \backslash F .
$$

As already mentioned, the requirement that $f^{n}$ be defined is always satisfied if $f$ is rational, and hence it can be (and of course always is) omitted from the definition. An analogous remark applies to transcendental entire functions, where $f^{n}$ is defined for all $z \in \mathbb{C}$. In this case, we always have $\infty \in J$.

A similar case is given by meromorphic functions with exactly one pole if this pole is an omitted value. (A complex number $z_{0}$ is called an omitted value of the meromorphic function $f$, if $f(z) \neq z_{0}$ for all $z \in \mathbb{C}$.) In this case, if the pole of $f$ is denoted by $z_{0}$, we have $\left\{z_{0}, \infty\right\} \subset J$, and $f^{n}(z)$ is defined for all $z \in \widehat{\mathbb{C}} \backslash\left\{z_{0}, \infty\right\}$.

It is not difficult to show that $f$ has the form

$$
f(z)=z_{0}+\frac{e^{g(z)}}{\left(z-z_{0}\right)^{m}}
$$

for some positive integer $m$ and some entire function $g$ in this case. It is no loss of generality to assume that $z_{0}=0$ so that

$$
f(z)=\frac{e^{g(z)}}{z^{m}}
$$

because otherwise we may consider $\phi^{-1}(f(\phi(z)))$ instead of $f$, where $\phi(z)=$ $z+z_{0}$. More generally, instead of maps of the form (1), we may consider analytic self-maps of $\mathbb{C} \backslash\{0\}$ here, without requiring that 0 be a pole of the map; compare [19, 36, 91, 92, 94, 95, 104, 114]. We shall restrict ourselves, however, to the case where $f$ is meromorphic in $\mathbb{C}$, our main interest being in the case where $f$ is entire or has several poles anyway.

In the remaining case, where $f$ has either at least two poles or only one pole which is not an omitted value, there are infinitely many points that are mapped onto a pole of $f$ by some iterate of $f$. For $z_{0} \in \widehat{\mathbb{C}}$, we define the backward orbit $O^{-}\left(z_{0}\right)$ of $z_{0}$ by

$$
O^{-}\left(z_{0}\right)=\bigcup_{n \geq 0} f^{-n}\left(z_{0}\right),
$$

where $f^{-n}\left(z_{0}\right)=\left\{z: f^{n}(z)=z_{0}\right\}$. Then the above statement is equivalent to saying that $O^{-}(\infty)$ is an infinite set. In fact, already $f^{-3}(\infty)$ is infinite, as follows easily from Picard's theorem. The largest open set where all iterates are defined is given by $\widehat{\mathbb{C}} \backslash \overline{O^{-}(\infty)}$. Since $f\left(\widehat{\mathbb{C}} \backslash \overline{O^{-}(\infty)}\right) \subset \widehat{\mathbb{C}} \backslash \overline{O^{-}(\infty)}$ and since $O^{-}(\infty)$ has more than two elements, $\left\{f^{n}\right\}$ is normal in $\widehat{\mathbb{C}} \backslash \overline{O^{-}(\infty)}$ by Montel's theorem. Hence

$$
F=\widehat{\mathbb{C}} \backslash \overline{O^{-}(\infty)} \text { and } \quad J=\overline{O^{-}(\infty)}
$$

compare [21] and [114]. We see that in this case the requirement that $\left\{f^{n}\right\}$ be normal can be omitted from the definition.

From this point of view, the iteration theory of entire functions and of meromorphic functions with one pole which is an omitted value is quite different from that of general meromorphic functions, which have at least two poles or 
only one pole which is not omitted. In the first two cases, it is clear where the iterates are defined, and we ask where they form a normal family. In the third case, we just ask where they are defined, and this then implies that they form a normal family there. Therefore, it is not surprising that there are major differences between these cases. On the other hand, there are also many analogies, but even here the proofs are often quite different.

According to the above remarks we shall divide the class of transcendental meromorphic functions for further reference into three subclasses:

- $E=\{f: f$ is transcendental entire $\}$;

- $P=\{f: f$ is transcendental meromorphic, has exactly one pole, and this pole is an omitted value $\}$;

- $M=\{f: f$ is transcendental meromorphic and has either at least two poles or exactly one pole which is not an omitted value $\}$.

Here $E$ and $M$ are thought of as mnemonics for entire and (general) meromorphic functions, while $P$ stands for one pole (or punctured plane). As already mentioned, we may (and often will) assume that functions in $P$ have the form (1).

2.2. Elementary properties of Fatou and Julia sets. By definition, $F$ is open and $J$ is closed. The properties of $F$ and $J$ contained in the following three lemmas are also easily verified, the proofs for transcendental functions being analogous to those for the rational case.

Lemma 1. If $f$ is rational, $f \in P$, or $f \in E$, then $F(f)=F\left(f^{n}\right)$ and $J(f)=$ $J\left(f^{n}\right)$ for all $n \geq 2$.

Here we have to exclude $f \in M$, because then $f^{n}$ is not meromorphic in $\mathbb{C}$ so that $F\left(f^{n}\right)$ and $J\left(f^{n}\right)$ are not defined. (There is, of course, a natural way to define $F\left(f^{n}\right)$ for $f \in M$ and $n \geq 2$, or, more generally, to define $F(f)$ for functions $f$ meromorphic in $\mathbb{C}$ except for countably many points. Then the conclusion of Lemma 1 holds for such functions.)

Lemma 2. $F$ and $J$ are completely invariant.

Here, by definition, a set $S$ is called completely invariant if $z \in S$ implies that $f(z) \in S$, unless $f(z)$ is undefined, and that $w \in S$ for all $w$ satisfying $f(w)=z$.

Lemma 3. Either $J=\widehat{\mathbb{C}}$ or $J$ has empty interior.

We note that the case $J=\widehat{\mathbb{C}}$ is actually possible. Examples of rational functions with this property are given by the rational functions that come from the multiplication theorems of elliptic functions. Usually, Lattès ([98], see also [90]) is credited with having introduced them into the subject, but it should perhaps be mentioned that already Böttcher [38, p. 63] was aware of these examples. For other examples of rational functions satisfying $J=\widehat{\mathbb{C}}$ we refer to $[27, \S 9.4, \S 11.9]$. The first example of an entire function with this property was given by Baker [11], who proved that $J\left(\lambda z e^{z}\right)=\widehat{\mathbb{C}}$ for a suitable value of $\lambda$. Later, Misiurewicz [109] proved that $J\left(e^{z}\right)=\widehat{\mathbb{C}}$, confirming a conjecture of Fatou [72, p. 370]. To obtain an example in $P$, we note that modifications of Baker's argument show that $J\left(\lambda e^{z} / z\right)=\widehat{\mathbb{C}}$ for a suitable value of $\lambda$. Finally, 
as an example in $M$, we mention that $J(\lambda \tan z)=\widehat{\mathbb{C}}$ for suitable values of $\lambda$. We comment on these examples in $\S 4.8$. We note that while $J=\widehat{\mathbb{C}}$ is possible, we always have $F \neq \widehat{\mathbb{C}}$. In fact, as we shall see in $\S 3.3, J$ is a perfect (and hence uncountable) set.

We say that $z_{0}$ is exceptional if $\mathrm{O}^{-}\left(z_{0}\right)$ is finite. It is not difficult to see that meromorphic functions have at most two exceptional values. (For transcendental functions this is an immediate consequence of Picard's theorem.) For rational functions the exceptional values are in the Fatou set. This is not necessarily the case for transcendental meromorphic functions. If $f \in P$ with pole at $z_{0}$, then $z_{0}$ and $\infty$ are exceptional, but there are no further exceptional values. Similarly, if $f \in E$, then $\infty$ is always exceptional so that there is at most one finite exceptional value. If a function $f \in E$ has a finite exceptional value $z_{0}$, then it has the form $f(z)=z_{0}+\left(z-z_{0}\right)^{m} e^{g(z)}$ for some nonnegative integer $m$ and an entire function $g$ so that $f$ can also be considered as a self-map of the punctured plane.

A simple consequence of Montel's theorem is the following result.

Lemma 4. If $z_{0} \in J$ is not exceptional, then $J=\overline{O^{-}\left(z_{0}\right)}$.

Similar to the backward orbit $\mathrm{O}^{-}\left(z_{0}\right)$, we define the forward orbit $\mathrm{O}^{+}\left(z_{0}\right)$ of $z_{0} \in \widehat{\mathbb{C}}$ by $O^{+}\left(z_{0}\right)=\bigcup_{n>0} f^{n}\left(z_{0}\right)$. Of course, here the union is taken only over those $n \geq 0$ for which $f^{n}\left(z_{0}\right)$ is defined. The orbit $O\left(z_{0}\right)$ of $z_{0}$ is defined by $O\left(z_{0}\right)=O^{+}\left(z_{0}\right) \cup O^{-}\left(z_{0}\right)$. For a subset $S$ of $\widehat{\mathbb{C}}$, we put $O^{ \pm}(S)=\bigcup_{z \in S} O^{ \pm}(z)$ and $O(S)=\bigcup_{z \in S} O(z)$. With this terminology, Lemma 2 may be written in the form $O(F) \subset F$ and $O(J) \subset J$.

Another simple consequence of Montel's theorem is that if $U$ is an open set that contains a point of $J$, then $\widehat{\mathbb{C}} \backslash O^{+}(U)$ contains at most two points, and these points are exceptional. For rational $f$, this implies that $O^{+}(J \cap U)=J$ and even that $f^{n}(J \cap U)=J$ for all sufficiently large $n$; see [27, Theorem 4.2.5]. For transcendental $f$ we find that $J \backslash O^{+}(J \cap U)$ contains at most two points and that these points are exceptional. However, we cannot deduce from this that $J \backslash f^{n}(J \cap U)$ contains only two points for sufficiently large $n$. In general, $J \backslash f^{n}(J \cap U)$ will contain neighborhoods of the exceptional points (if they exist). But if $f \in M$ does not have exceptional points or if they are in $F$, then $f^{n}(J \cap U)=J$ for all sufficiently large $n$.

\section{PERIODIC POINTS}

3.1. Definitions. An important role in iteration theory is played by the periodic points. By definition, $z_{0}$ is called a periodic point of $f$ if $f^{n}\left(z_{0}\right)=z_{0}$ for some $n \geq 1$. In this case, $n$ is called a period of $z_{0}$, and the smallest $n$ with this property is called the minimal period of $z_{0}$. For a periodic point $z_{0}$ of minimal period $n,\left(f^{n}\right)^{\prime}\left(z_{0}\right)$ is called the multiplier of $z_{0}$. (If $z_{0}=\infty$, which can happen only for rational function $f$, of course, this has to be modified. In this case, the multiplier is defined to be $\left(g^{n}\right)^{\prime}(0)$ where $g(z)=1 / f(1 / z)$.) A periodic point is called attracting, indifferent, or repelling accordingly as the modulus of its multiplier is less than, equal to, or greater than 1. Periodic points of multiplier 0 are called superattracting. (Some writers reserve the term attracting for the case $0<\left|\left(f^{n}\right)^{\prime}\left(z_{0}\right)\right|<1$, but we consider superattracting as a special case of attracting.) The multiplier of an indifferent periodic point 
is of the form $e^{2 \pi i \alpha}$ where $0 \leq \alpha<1$. We say that $z_{0}$ is rationally indifferent if $\alpha$ is rational and irrationally indifferent otherwise. Also, a point $z_{0}$ is called preperiodic if $f^{n}\left(z_{0}\right)$ is periodic for some $n \geq 1$. Finally, a periodic point of period 1 is called a fixed point.

It is easy to see that attracting periodic points are in $F$, while repelling and rationally indifferent periodic points are in $J$. For irrationally indifferent periodic points the question whether they are in $F$ or $J$ is difficult to decide. Both possibilities do occur. We refer the reader to the classical papers of Cremer [47, 49] and Siegel [119], as well as more recent work of Yoccoz [138, 139]. An exposition of these and other results, together with further references, can be found in [113].

The behavior of the iterates in the neighborhood of a fixed point (or, more generally, a periodic point) is intimately connected with the solution of certain functional equations. For most results in this direction, it is required only that the function under consideration is defined in a neighborhood of the fixed point, and it is usually irrelevant whether it extends to a rational or transcendental meromorphic function. Therefore, we omit this topic here but refer to the papers and books on iteration of rational functions cited in the introduction.

3.2. Existence of periodic points. It is clear that a rational function has periodic points of (not necessarily minimal) period $n$ for all $n \geq 1$. Transcendental entire functions, however, need not have fixed points, that is, periodic points of period 1. A simple example is given by $f(z)=e^{z}+z$. On the other hand, already Fatou [72, p. 345] proved that an entire transcendental function $f$ has at least one periodic point of period 2. The idea is to consider the function

$$
h(z)=\frac{f(f(z))-z}{f(z)-z} .
$$

If $f$ does not have periodic points of period 2 (and hence does not have fixed points), then $h$ is an entire function which does not take the values 0 and 1 . By Picard's theorem $h$ is constant. Once this is known, it is not difficult to obtain a contradiction. Fatou's result was generalized by Rosenbloom [117], who proved the following theorem.

Theorem 1. An entire transcendental function has infinitely many periodic points of period $n$ for all $n \geq 2$.

The idea of the proof is similar. Instead of Picard's theorem, however, Rosenbloom used something stronger, namely, Nevanlinna's theory on the distribution of values, which may be considered as a quantitative version of Picard's theorem. Since this is the only place in this paper where we use Nevanlinna theory, we do not give an introduction to it but refer to $[79,87,111]$ for notation and basic results.

To prove Theorem 1, we suppose that $f^{n}$ and hence $f$ have only finitely many fixed points and consider the auxiliary function $h$ defined by

$$
h(z)=\frac{f^{n}(z)-z}{f^{n-1}(z)-z} .
$$


Then

$$
\begin{aligned}
N(r, h) & =O\left(T\left(r, f^{n-1}\right)\right), \\
N\left(r, \frac{1}{h}\right) & =O(\log r),
\end{aligned}
$$

and

$$
N\left(r, \frac{1}{h-1}\right)=O\left(T\left(r, f^{n-1}\right)\right)
$$

as $r \rightarrow \infty$. Also, it is not difficult to prove that $T\left(r, f^{n-1}\right)=o\left(T\left(r, f^{n}\right)\right)$ as $r \rightarrow \infty$ outside some exceptional set of finite measure; see [87, p. 147]. (In fact, this last result even holds without exceptional set; see [45, Theorems 1 and 2] or [79, Lemma 2.6].) We deduce that $T(r, h) \sim T\left(r, f^{n}\right)$ and hence that

$$
N(r, h)+N\left(r, \frac{1}{h}\right)+N\left(r, \frac{1}{h-1}\right)=o(T(r, h))
$$

as $r \rightarrow \infty$ outside the exceptional set. This contradicts Nevanlinna's second fundamental theorem. Thus the proof of Theorem 1 is complete.

One may ask whether there is some quantitative version of Theorem 1 in the sense that there is a lower bound for $N\left(r, 1 /\left(f^{n}(z)-z\right)\right)$ in terms of $T\left(r, f^{n}\right)$ if $n \geq 2$ and $f \in E$. Denote by $\delta(a, h)$ the deficiency of a meromorphic $h$ with respect to the value $a \in \widehat{\mathbb{C}}$.

Question 1. Do we have $\delta\left(0, f^{n}(z)-z\right)=0$ (or at least $\delta\left(0, f^{n}(z)-z\right)<1$ ) if $f \in E$ and $n \geq 2$ ?

Some (much weaker) results of this type can be found in $[4,5,28,136]$.

If $f \in P$, then the conclusion of Theorem 1 is true even if $n=1$. To see this, suppose $f \in P$ and define $F(z)=f(z) / z$. Then $F$ has no zeros and at most two poles. Hence, by Picard's theorem $F$ takes the value 1 infinitely often, that is, $f$ has infinitely many fixed points. If $f \in M$, then $f$ need not have fixed points; consider $f(z)=z+1 / g(z)$ where $g$ is entire transcendental. But we shall see below that $f$ has infinitely many periodic points of period $n$ if $n \geq 2$.

Baker $[4-6,8]$ seems to have been the first who addressed the question in which cases a rational or entire function may fail to have periodic points of minimal period $n$ for some $n$. He proved [8] that if a rational function $f$ of degree $d \geq 2$ has no periodic point of minimal period $n$, then $n=2$ and $d \in\{2,3,4\}$ or $n=3$ and $d=2$. Moreover, if $f$ is a polynomial, then only the case $n=d=2$ can occur. Earlier he had proved [6] that if $f$ is an entire function, then there exists at most one integer $n \geq 1$ (depending on $f$ ) such that $f$ does not have periodic points of minimal period $n$.

The latter result can be strengthened as follows.

Theorem 2. If $f$ is a transcendental meromorphic function and $n \geq 2$, then $f$ has infinitely many periodic points of minimal period $n$.

As already mentioned, this also holds for $n=1$ if $f \in P$, but not in general if $f \in E$ or $f \in M$.

Theorem 2 was proved in [29] if $f \in E$ and in [36, Chapter 5.2] if $f \in P$. A proof for $f \in M$, using the ideas of [30], is as follows. 
Suppose first that $f^{n-1}$ has at least three poles $p_{1}, p_{2}, p_{3}$. Define $g=f^{n-1}$ and denote by $m_{j}$ the order of the pole $p_{j}$. There exist functions $h_{j}$, defined and analytic in a neighborhood of 0 , such that $g\left(p_{j}+h_{j}(z)\right)=z^{-m_{j}}$. Define $k_{1}(z)=p_{1}+h_{1}\left(z^{m_{2} m_{3}}\right), k_{2}(z)=p_{2}+h_{2}\left(z^{m_{1} m_{3}}\right)$, and $k_{3}(z)=p_{3}+h_{3}\left(z^{m_{1} m_{2}}\right)$ so that $g\left(k_{j}(z)\right)=z^{-m_{1} m_{2} m_{3}}$. Suppose now that $f$ does not have periodic points of period $n$ in neighborhoods of $p_{1}, p_{2}, p_{3}$. Then $F(z)=f\left(z^{-m_{1} m_{2} m_{3}}\right)=$ $f^{n}\left(k_{j}(z)\right) \neq k_{j}(z)$ in a neighborhood of 0 . Hence

$$
\frac{\left(F(z)-k_{1}(z)\right)\left(k_{3}(z)-k_{2}(z)\right)}{\left(F(z)-k_{2}(z)\right)\left(k_{3}(z)-k_{1}(z)\right)} \neq 0,1, \infty
$$

in a neighborhood of 0 , contradicting Picard's theorem. Hence the periodic points of period $n$ must accumulate at $p_{1}, p_{2}$, or $p_{3}$. Since periodic points of period $k$ where $1 \leq k \leq n-1$ cannot accumulate at poles of $f^{n-1}$, we deduce that $f$ has infinitely many periodic points of minimal period $n$.

The case that $f^{n-1}$ has exactly two poles is similar. Here we choose $k_{1}(z)=$ $p_{1}+h_{1}\left(z^{m_{2}}\right), k_{2}(z)=p_{2}+h_{2}\left(z^{m_{1}}\right), F(z)=f\left(z^{-m_{1} m_{2}}\right)$ and observe that

$$
\frac{F(z)-k_{1}(z)}{F(z)-k_{2}(z)} \neq 0,1, \infty
$$

in a neighborhood of 0 .

Finally, we consider the case that $f^{n-1}$ has only one pole but is in $M$. Then necessarily $n=2$ or $n=3$. We leave it to the reader to check that the method of [29] can be extended to this case.

It is perhaps worth mentioning (and, at least for me, surprising) that the proof given above for functions with at least two poles is shorter and more elementary than the proofs for the classes $E$ and $P$ so that these questions are much simpler for functions with poles than for entire functions. We will give a generalization of Theorem 2 in $\S 3.4$. Its proof, however, will be less elementary (but still short).

3.3. The Julia set is perfect. The results concerning the existence of periodic points may be used to prove that $J \neq \emptyset$. More generally, we have the following result.

Theorem 3. Let $f$ be a meromorphic function. Then $J(f)$ is perfect.

Recall that a set is called perfect if it is closed, nonempty, and does not contain isolated points.

We first prove that $J$ is not empty and in fact an infinite set. There are essentially two ways to do this if $f$ is rational. One method is to assume that $f^{n_{j}} \rightarrow \phi$ uniformly in $\widehat{\mathbb{C}}$. Then $\phi$ must also be rational and $\operatorname{deg}(\phi)=\lim _{j \rightarrow \infty} \operatorname{deg}\left(f^{n_{j}}\right)$. But $\operatorname{deg}\left(f^{n_{j}}\right)=(\operatorname{deg}(f))^{n_{j}} \rightarrow \infty$ as $j \rightarrow \infty$, provided $\operatorname{deg}(f) \geq 2$, a contradiction. The other method (which is the one used by Fatou and Julia) is to prove that $f$ has a fixed point which is repelling or has multiplier 1 . Once a point $z_{0} \in J$ is found, it is not difficult to see that $O^{-}\left(z_{0}\right)$ is infinite. Hence $J$ is infinite because $O^{-}\left(z_{0}\right) \subset J$.

Once this is known, we can prove that $J$ is perfect as follows. Suppose that $w_{0} \in J$, and let $N$ be a neighborhood of $w_{0}$. We can find $w_{1}, w_{2}, w_{3} \in$ $J \backslash O^{+}\left(w_{0}\right)$. Because $\left\{\left.f^{n}\right|_{N}\right\}$ is not normal, $w_{j} \in O^{+}(N)$ for some $j \in\{1,2,3\}$. 
Hence $O^{-}\left(w_{j}\right) \cap N \backslash\left\{w_{0}\right\}$ is not empty. In particular, $J \cap N \backslash\left\{w_{0}\right\}$ is not empty. Hence $w_{0}$ is not isolated, that is, $J$ is perfect.

Both methods to prove that $J$ is infinite do not generalize to the case where $f$ is transcendental (see, however, the discussion of the second method in the next section). On the other hand, it is clear from the discussion in $\S 2.1$ that $J(f)$ is an infinite and, in fact, a perfect set if $f \in M$.

We sketch how one can prove that $J(f)$ is infinite in the case that $f \in E$ or $f \in P$. First we note that we may assume $f$ has infinitely many fixed points. As already mentioned above, this is always the case if $f \in P$. And if $f \in E$, then we may consider $f^{2}$ instead of $f$ because of Lemma 1 , and $f^{2}$ always has infinitely many fixed points by Theorem 1 . If infinitely many of the fixed points of $f$ are in $J$, then we are done. Hence we may assume that there exist two fixed points $p$ and $q$ of $f$ that are contained in $F$. If $p$ and $q$ are in different components of $F$, then any path connecting them must meet $J$, and we are also done. Thus we may assume that there exists a component $U$ of $F$ which contains $p$ and $q$. Clearly, the limit functions of $\left\{\left.f^{n}\right|_{U}\right\}$ cannot be constant. We deduce that if $f^{n_{j}}(z) \rightarrow \phi(z)$ for $z \in U$ as $j \rightarrow \infty$, then $f^{n_{j+1}-n_{j}}(z) \rightarrow z$ for $z \in U$. This implies that $\left.f\right|_{U}$ is an automorphism of $U$. Hence $f^{-1}(U)$ contains components of $F$ different from $U$. Again, any path connecting these components meets $J$. This completes the proof that $J$ is an infinite set. (With a little more effort one can show that a component of $F$ cannot contain two fixed points; see [27, Lemma 6.9.3], and compare also Theorem 6 in $\S 4.2$.) The proof that $J$ is perfect can now be carried out as in the rational case.

3.4. Julia's approach. So far our development of the theory has followed Fatou's ideas. Julia based his theory on the closure of the set of repelling periodic points. One of the basic results of the theory is that these two sets are actually equal.

Theorem 4. Let $f$ be a meromorphic function. Then $J(f)$ is the closure of the set of repelling periodic points of $f$.

For rational $f$, this result was obtained by both Fatou [71, $\S 30$, p. 69] and Julia [89, p. 99, p. 118]. Their proofs, however, were different. (A good exposition of both proofs can be found in [108, §11].) Fatou proved first that any point in $J$ is the limit point of periodic points and then that there are only finitely many nonrepelling periodic points, which together implies the result. The first part does carry over to transcendental functions ([72, p. 354], see also [44]), but the second part clearly does not, as can be seen by simple examples like $f(z)=e^{z}+z+1$. For Julia's method it is essential that the set of repelling periodic points is not empty. In fact, it suffices that the set of repelling and rationally indifferent periodic points is not empty, and a rational function always has at least one fixed point which is repelling or has multiplier 1 (see [71, $\S 2$, p. 168] and [89, p. 85, p. 243]). Julia's method does carry over to transcendental functions with a repelling or rationally indifferent fixed point which is not exceptional (this is done in [57, pp. 229-230; 59, pp. 69-70], but in general transcendental functions need not have such a fixed point; in fact, they need not have fixed points at all. 
Baker [10] proved that Theorem 4 holds for entire transcendental functions as well. His proof was based on a deep theorem of Ahlfors [2, 79, 111] from his theory of covering surfaces. Theorem 4 was extended to class $P$ in [36, Theorem 5.2] and to class $M$ in [21, Theorem 1], the proofs being based again on Ahlfors's theorem.

We sketch the argument for class $M$ and begin with the statement of a version of Ahlfors's theorem. (A different version is used in [10] and [36].) Unfortunately, lack of space prevents us from discussing the proof of this important result.

Lemma 5. Let $f$ be a transcendental meromorphic function, and let $D_{1}, D_{2}$, $\ldots, D_{5}$ be five simply connected domains in $\mathbb{C}$ with disjoint closures. Then there exists $j \in\{1,2, \ldots, 5\}$ and, for any $R>0$, a simply connected domain $G \subset\{z \in \mathbb{C}:|z|>R\}$ such that $f$ is a conformal map of $G$ onto $D_{j}$. If $f$ has only finitely many poles, then "five" may be replaced by "three".

Following [21], we deduce the following result.

Lemma 6. Suppose that $f \in M$ and that $z_{1}, z_{2}, \ldots, z_{5} \in O^{-}(\infty) \backslash\{\infty\}$ are distinct. Define $n_{j}$ by $f^{n_{j}}\left(z_{j}\right)=\infty$. Then there exists $j \in\{1,2, \ldots, 5\}$ such that $z_{j}$ is a limit point of repelling periodic points of minimal period $n_{j}+1$. If $f$ has only finitely many poles, then "five" may be replaced by "three".

To deduce Lemma 6 from Lemma 5, we choose the $D_{j}$ as discs around $z_{j}$ where the radii are chosen so small that the $D_{j}$ do not contain critical points of $f$ and that their closures are pairwise disjoint. There exists $R>0$ such that $f^{n_{j}}\left(D_{j}\right) \supset\{z:|z|>R\} \cup\{\infty\}$. We choose $j$ and $G$ according to Lemma 5 . Then we can find $H \subset D_{j}$ such that $f^{n_{j}}: H \rightarrow G$, and hence $f^{n_{j}+1}: H \rightarrow D_{j}$ is a conformal mapping. Moreover, $\bar{H} \subset D_{j}$, and this implies that the inverse function $f^{-n_{j}-1}$ of $f^{n_{j}+1}: H \rightarrow D_{j}$ has an attracting fixed point in $D_{j}$. Clearly, this attracting fixed point of $f^{-n_{j}-1}$ is a repelling periodic point of $f$ of period $n_{j}+1$. Because the $D_{j}$ can be chosen arbitrarily small, the repelling periodic points of period $n_{j}+1$ accumulate at $z_{j}$. Because $z_{j}$ is a pole of $f^{n_{j}}$, periodic points of period less than $n_{j}+1$ cannot accumulate at $z_{j}$; hence, $z_{j}$ is a limit point of repelling periodic points of minimal period $n_{j}+1$. This completes the proof of Lemma 6.

Lemma 6 yields immediately that the conclusion of Theorem 4 holds for $f \in M$ because $J=\overline{O^{-}(\infty)}$ is perfect.

Another interesting consequence of Lemma 6 is that if $f^{-n+1}(\infty)$ contains more than four elements, then $f$ has infinitely many repelling periodic points of minimal period $n$. In particular, this is the case if $f \in M$ and $n \geq 4$. We also see that $f$ has infinitely many repelling periodic points of minimal period 2 and 3 if $f$ has more than two poles. On the other hand, it was proved in [29] that if $f$ is entire transcendental, then $f$ has infinitely many repelling periodic points of minimal period $n$ for all $n \geq 2$. The method used there can be extended to the case where $n=2$ or $n=3$ and $f$ has one or two poles. Hence we obtain the following generalization of Theorem 2 .

Theorem 5. If $f$ is a transcendental meromorphic function and $n \geq 2$, then $f$ has infinitely many repelling periodic points of minimal period $n$. 
We remark that in view of Lemma 1, Julia's method can be used to obtain Theorem 4 from Theorem 5. This does not, however, constitute a new proof of Theorem 4, because the argument in [29] also uses Ahlfors's theorem. The fact that all known proofs of the existence of repelling periodic points are based on this deep result makes Julia's approach to start with the closure of the set of repelling periodic points inadequate for transcendental functions, because it is difficult to see that this set is not empty.

It would be of interest to give a more elementary proof of Theorem 4 .

Question 2. Is there a proof of Theorem 4 which does not use Ahlfors's result?

As already mentioned, it suffices to prove the existence of a repelling or rationally indifferent periodic point which is not exceptional.

\section{The Components of the Fatou SeT}

4.1. The types of domains of normality. Let $U$ be a (maximal) domain of normality of the iterates of $f$, that is, a component of $F$. (Here and in the following, "component" always means "connected component".) Then $f^{n}(U)$ is contained in a component of $F$ which we denote by $U_{n}$. A component $U$ is called preperiodic if there exist $n>m \geq 0$ such that $U_{n}=U_{m}$. In particular, if this is the case for $m=0$ (where $U_{0}=U$ ) and some $n \geq 1$, then $U$ is called periodic with period $n$, and $\left\{U, U_{1}, \ldots, U_{n-1}\right\}$ is called a (periodic) cycle of components. Again, the smallest $n$ with this property is called the minimal period of $U$. In the case $n=1$, that is, if $f(U) \subset U, U$ is called invariant. A component of $F$ which is not preperiodic is called a wandering component (or wandering domain).

For rational functions, we have $f(U)=U_{1}$, but for transcendental functions it is possible that $f(U) \neq U_{1}$. For example, if $f(z)=\lambda e^{z}$ where $0<\lambda<e^{-1}$, then $F$ consists of a single component which contains 0 , but clearly $0 \notin f(F)$. Similarly, if $f(z)=\lambda \tan z$ where $0<\lambda<1$, then $F$ consists of a single component which contains $\pm \lambda i$, but $\pm \lambda i \notin f(F)$.

Values in $U_{1} \backslash f(U)$ need not be omitted values. As an example, we consider $f(z)=z \exp \left(\left(-z^{2}+3 z-2\right) / 6\right)$. Then 0 and 2 are attracting fixed points, while 1 is a repelling fixed point. Let $V$ be the component of $F$ that contains 0 . All large positive real numbers are contained in a component $U$ satisfying $f(U) \subset V$; that is, we have $V=U_{1}$. It is not difficult to show that $U \neq V$. (For instance, this follows from the fact that $U$ and $V$ are simply connected and symmetric with respect to the real axis.) It follows that $0 \in U_{1} \backslash f(U)$.

On the other hand, it is easy to see that values in $U_{1} \backslash f(U)$ are asymptotic values of $f$, the asymptotic path being contained in $U$. As pointed out in [21, p. 242], one can deduce from Gross's star theorem that $f(U)$ is a dense open subset of $U_{1}$. If $f \in E$, then $U_{1} \backslash f(U)$ contains at most one point. I. N. Baker has kindly informed me that this result was proved by M. Herring. It had also been obtained independently in [34].

Question 3. Can $U_{1} \backslash f(U)$ have more than two points if $f \in M$ ?

4.2. The classification of periodic components. The behavior of $f^{n}$ in periodic components is well understood. 
Theorem 6. Let $U$ be a periodic component of period $p$. Then we have one of the following possibilities:

- $U$ contains an attracting periodic point $z_{0}$ of period $p$. Then $f^{n p}(z) \rightarrow$ $z_{0}$ for $z \in U$ as $n \rightarrow \infty$, and $U$ is called the immediate attractive basin of $z_{0}$.

- $\partial U$ contains a periodic point $z_{0}$ of period $p$ and $f^{n p}(z) \rightarrow z_{0}$ for $z \in U$ as $n \rightarrow \infty$. Then $\left(f^{p}\right)^{\prime}\left(z_{0}\right)=1$ if $z_{0} \in \mathbb{C}$. (For $z_{0}=\infty$ we have $\left(g^{p}\right)^{\prime}(0)=1$ where $g(z)=1 / f(1 / z)$.) In this case, $U$ is called $a$ Leau domain.

- There exists an analytic homeomorphism $\phi: U \rightarrow D$ where $D$ is the unit disc such that $\phi\left(f^{p}\left(\phi^{-1}(z)\right)\right)=e^{2 \pi i \alpha} z$ for some $\alpha \in \mathbb{R} \backslash \mathbb{Q}$. In this case, $U$ is called a Siegel disc.

- There exists an analytic homeomorphism $\phi: U \rightarrow A$ where $A$ is an annulus, $A=\{z: 1<|z|<r\}, r>1$, such that $\phi\left(f^{p}\left(\phi^{-1}(z)\right)\right)=e^{2 \pi i \alpha} z$ for some $\alpha \in \mathbb{R} \backslash \mathbb{Q}$. In this case, $U$ is called a Herman ring.

- There exists $z_{0} \in \partial U$ such that $f^{n p}(z) \rightarrow z_{0}$ for $z \in U$ as $n \rightarrow \infty$, but $f^{p}\left(z_{0}\right)$ is not defined. In this case, $U$ is called a Baker domain.

Clearly, if $f$ is rational, then Baker domains do not exist. If $f \in E$, then Baker domains are possible only for $z_{0}=\infty$. Similarly, if $f \in P$ with pole at 0 , then Baker domains are possible only for $z_{0} \in\{0, \infty\}$.

The above classification theorem is essentially due to Cremer [48] and Fatou [71]. Fatou [71, $\S 56$, p. 249] proved that if $\left\{\left.f^{n}\right|_{U}\right\}$ has only constant limit functions, then $U$ is an immediate attractive basin or a Leau domain, provided $f$ is rational. His proof shows that the only further possibility in the case of transcendental functions is that of a Baker domain. Cremer [48, p. 317] proved that if $\left\{\left.f^{n}\right|_{U}\right\}$ has nonconstant limit functions, then $U$ is a Siegel disc or a Herman ring. Neither Fatou nor Cremer stated the full classification theorem, but Töpfer's remarks [134, p. 211] come fairly close to it.

We remark that when Fatou and Cremer wrote their papers, it was not known yet that Siegel discs and Herman rings do actually exist, and they may have believed that such domains do not exist. (Cremer [47, p. 154] wrote that it is conjectured that rational functions do not have Siegel discs but also that he does not see a reason for this conjecture.) Töpfer knew about Siegel discs, but the existence of Herman rings (which Töpfer called "Zentrumring") was not established yet.

In the above form the classification theorem was stated first by Baker, Kotus, and $L \ddot{u}$ [21, Theorems 2.2 and 2.3]. In the case of rational functions it seems to have been given first by Sullivan [129, 131]. We remark that the case of an immediate attractive basin is sometimes further distinguished depending on whether the attracting periodic point contained in it is superattracting or not. If this is the case, then $U$ is called a Bötcher domain; otherwise, $U$ is called a Schröder domain. The other notations are also not uniform in the literature: Leau domains are also called parabolic domains; Herman rings are also named after Arnol'd; and for Baker domains the names infinite Fatou component [82], essentially parabolic domain [24], and domains at $\infty$ [59] are also used. The term "Baker domain" seems to have been used first in $[69,70]$.

Besides the papers cited already, we refer to $[27,108,127]$ for a proof of the 
classification theorem. Here only the case that $f$ is rational is considered, but the changes necessary to handle the case that $f$ is transcendental are minor.

We note that if $f$ is entire, then $f$ does not have Herman rings. In fact, a simple argument shows that $f^{n} \rightarrow \infty$ in multiply connected components of $F$; see [133, p. 67]. Baker [19, Theorems 1 and 3] proved that analytic self-maps of the punctured plane may have a Herman ring but at most one (which has period 1). It is not clear whether this is possible for $f \in P$.

4.3. The role of the singularities of the inverse function. The periodic domains are closely related to the set of singularities of the inverse function $f^{-1}$ of $f$, that is, the set of critical and finite asymptotic values of $f$ and (finite) limit points of these values. Denote this set by $\operatorname{sing}\left(f^{-1}\right)$.

Theorem 7. Let $f$ be a meromorphic function, and let $C=\left\{U_{0}, U_{1}, \ldots, U_{p-1}\right\}$ be a periodic cycle of components of $F$.

- If $C$ is a cycle of immediate attractive basins or Leau domains, then $U_{j} \cap$ $\operatorname{sing}\left(f^{-1}\right) \neq \emptyset$ for some $j \in\{0,1, \ldots, p-1\}$. More precisely, there exists $j \in\{0,1, \ldots, p-1\}$ such that $U_{j} \cap \operatorname{sing}\left(f^{-1}\right) \neq \emptyset$ contains $a$ point which is not preperiodic or such that $U_{j}$ contains a periodic critical point (in which case $C$ is a cycle of superattractive basins).

- If $C$ is a cycle of Siegel discs or Herman rings, then $\partial U_{j} \subset \overline{O^{+}\left(\operatorname{sing}\left(f^{-1}\right)\right)}$ for all $j \in\{0,1, \ldots, p-1\}$.

These results were proved by Fatou [71, $\S 30-31]$ for rational maps, but the proofs extend to the transcendental case.

It follows from Theorem 7 that the number of cycles of immediate attractive basins and Leau domains does not exceed the number of singularities of $f^{-1}$. For transcendental functions, $\operatorname{sing}\left(f^{-1}\right)$ may of course be infinite (and simple examples like $f(z)=e^{z}+z+1$ or $f(z)=e^{z}+z+2$ show that there may, in fact, be infinitely many cycles of immediate attractive basins and Leau domains), but for a rational function $f$ of degree $d$ there are at most $2 d-2$ singularities of $f^{-1}$.

The number of cycles of Siegel discs and Herman rings of a rational function may also be bounded in terms of the degree $d$. The sharp bound is due to Shishikura [121, p. 5], who, strengthening earlier results of Fatou [71, §30] and Sullivan [131, p. 6], proved that the number of cycles of immediate attractive basins, Leau domains, and Siegel discs plus twice the number of cycles of Herman rings does not exceed $2 d-2$. Loosely speaking, cycles of immediate attractive basins, Leau domains, and Siegel discs require one critical point, while cycles of Herman rings need at least two. (Theorem 7 gives an heuristic argument of why this should be true but by no means proves it.) A result of Shishikura's type for a class of transcendental entire functions can be found in [70, Theorem 5].

One may ask whether Baker domains are also related to singularities of $f^{-1}$. Examples in [68, Example 3] and [82, p. 609] show that a periodic cycle of Baker domains need not contain points of $\operatorname{sing}\left(f^{-1}\right)$.

Question 4. Let $f$ be a meromorphic function with a cycle of Baker domains that does not contain a point of $\operatorname{sing}\left(f^{-1}\right)$. Is there some relation between $\operatorname{sing}\left(f^{-1}\right)$ and the boundaries of the domains of this cycle? 
We will see in Theorem 13 in $\S 4.7$ that if $f^{n} \nrightarrow \infty$ in a cycle of Baker domains, then some domain in this cycle has a finite asymptotic value on its boundary (regardless of whether there are singularities of $f^{-1}$ in this cycle or not). Question 4 asks whether more can be said if no points of $\operatorname{sing}\left(f^{-1}\right)$ are in this cycle. More specifically, one may ask the following question:

Question 5. Is it possible that a meromorphic function $f$ has Baker domains if $O^{+}(z)$ is bounded for all $z \in \operatorname{sing}\left(f^{-1}\right)$ ?

4.4. The connectivity of the components of the Fatou set. By definition, Siegel discs are simply connected and Herman rings are doubly connected. In this section we consider the connectivity of the other components of $F$.

Theorem 8. Let $f$ be a meromorphic function, and let $U$ be an invariant component of $F$. Then the connectivity of $U$ has one of the values 1,2 , or $\infty$. Here 2 occurs only when $U$ is a Herman ring.

This was proved by Fatou [71, §32] if $f$ is rational (see also [27, §7.5] for this case) and by Baker, Kotus, and Lü [23, Theorem 3.1] if $f \in M$. Of course, the result implies that the connectivity of a periodic component (of period greater than 1) also takes one of the values 1,2 , or $\infty$ if $f$ rational. Probably this remains true for functions in $M$ as well, but the proof in [23] does not seem to give this result.

Question 6. Let $f$ be a meromorphic function, and let $U$ be a periodic component of $F$. Is the connectivity of $U$ either 1,2 , or $\infty$ ?

Baker, Kotus, and Lü [23, Theorem 6.1] also proved that, in contrast to Theorem 8 , the connectivity of a preperiodic component may take any value if $f$ is rational or if $f \in M$. Moreover, they gave examples of functions in $M$ which have a wandering domain of any preassigned connectivity [22].

For functions in $P$ and $E$, we have results stronger than Theorem 8 . In fact, as proved by Baker [18, Theorem 1], the connectivity of any component of $F$ is 1 or 2 if $f$ is an analytic self-map of $\mathbb{C} \backslash\{0\}$ and, hence, in particular if $f \in P$. For entire functions we have the following result.

Theorem 9. If $f \in E$, then any preperiodic component of $F$ is simply connected.

In other words, multiply connected components of $F$ are necessarily wandering if $f \in E$.

Theorem 9 is an immediate consequence of a result of Baker [13, Theorem 1], who proved that multiply connected components of $F(f)$ are bounded if $f \in E$. In order to give a proof of Theorem 9 (following Baker's argument), we start with the following lemma. With further applications in mind, this lemma is stated in a form more general than needed for the proof of Theorem 9. The results contained in it can be found in [20, Lemmas 1 and 2; 23, Lemma 4.1] (see also [15, Theorem 6; 103, Proposition A.1]).

Lemma 7. Let $G$ be an unbounded open set in $\mathbb{C}$ with at least two finite boundary points, and let $g$ be analytic in $G$. Let $D$ be a domain contained in $G$, and suppose that $g^{n}(D) \subset G$ for all $n$ and that $\left.g^{n}\right|_{D} \rightarrow \infty$ as $n \rightarrow \infty$. Then, 
for any compact subset $K$ of $D$, there exist constants $C$ and $n_{0}$ such that

$$
\left|g^{n}\left(z^{\prime}\right)\right| \leq\left|g^{n}(z)\right|^{C}
$$

for all $z, z^{\prime} \in K$ and $n \geq n_{0}$. If, in addition, $g(D) \subset D$, then we also have

$$
\log \log \left|g^{n}(z)\right|=O(n)
$$

for all $z \in D$ as $n \rightarrow \infty$, and there exist a constant $A>1$ and a curve $\gamma \subset D$ tending to $\infty$ which satisfies $g(\gamma) \subset \gamma$ such that

$$
|z|^{1 / A} \leq|g(z)| \leq|z|^{A}
$$

for $z \in \gamma$. If $\widehat{\mathbb{C}} \backslash G$ contains a connected set $\Gamma$ such that $\{a, \infty\} \subset \Gamma$ for some $a \in \mathbb{C}$, then (2), (3), and (4) may be replaced by

$$
\begin{gathered}
\left|g^{n}\left(z^{\prime}\right)\right| \leq C\left|g^{n}(z)\right|, \\
\log \left|g^{n}(z)\right|=O(n),
\end{gathered}
$$

and

$$
\frac{|z|}{A} \leq|g(z)| \leq A|z| .
$$

In particular, this is the case if $G$ is simply connected.

In order to prove Lemma 7, we denote by $\Omega$ the plane punctured at two finite boundary points of $G$. By $\left[z, z^{\prime}\right]_{\Omega}$ we denote the hyperbolic distance of two points $z$ and $z^{\prime}$ in $\Omega$. To prove (2), we note that

$$
\left[g^{n}(z), g^{n}\left(z^{\prime}\right)\right]_{\Omega} \leq\left[g^{n}(z), g^{n}\left(z^{\prime}\right)\right]_{G} \leq\left[g^{n}(z), g^{n}(z)\right]_{g^{n}(D)} \leq\left[z, z^{\prime}\right]_{D}
$$

and that the hyperbolic metric $\rho_{\Omega}(z)$ satisfies

$$
\rho_{\Omega}(z) \sim \frac{c}{|z| \log |z|}
$$

for some positive constant $c$ as $|z| \rightarrow \infty$; see [3, §1.8]. It follows that if $\left|g^{n}\left(z^{\prime}\right)\right| \geq\left|g^{n}(z)\right|$, then

$$
\left[g^{n}(z), g^{n}\left(z^{\prime}\right)\right]_{\Omega} \geq \frac{c}{2} \int_{\left|g^{n}(z)\right|}^{\left|g^{n}\left(z^{\prime}\right)\right|} \frac{d t}{t \log t}=\frac{c}{2} \log \left(\frac{\log \left|g^{n}\left(z^{\prime}\right)\right|}{\log \left|g^{n}(z)\right|}\right)
$$

for sufficiently large $n$ so that (2) holds with

$$
C=\exp \left(\frac{2}{c} \max _{z, z^{\prime} \in K}\left[z, z^{\prime}\right]_{D}\right) .
$$

In order to prove (4), we choose $\sigma$ as a curve in $D$ that connects a point $z_{0} \in D$ with $g\left(z_{0}\right)$ and define $\gamma=\bigcup_{n=0}^{\infty} g^{n}(\sigma)$. Then (4) can be deduced from (2) if we choose $K=\sigma \cup g(\sigma)$. Similarly, choosing $z^{\prime}=g(z)$ in (2), we have

$$
\left|g^{n}(z)\right|=\left|g^{n-1}\left(z^{\prime}\right)\right| \leq\left|g^{n-1}(z)\right|^{C} \leq\left|g^{n-2}(z)\right|^{C^{2}} \leq \cdots
$$

for large $n$, and (3) follows by induction.

To prove (5)-(7), we proceed as above but define $\Omega$ as the component of $\widehat{\mathbb{C}} \backslash \Gamma$ that contains $G$. Then $\rho_{\Omega}(z) \geq c /|z|$ for some positive constant $c$ and 
all sufficiently large $z \in \Omega$. The arguments used above to prove (2)-(4) now yield (5)-(7). This completes the proof of Lemma 7.

We now prove Theorem 9. Suppose that $U$ is a multiply connected component of $F$, and let $\sigma \subset U$ be a curve that is not null-homotopic in $U$. Define $\sigma_{n}=f^{n}(\sigma)$. Then $\sigma_{n}$ is not null-homotopic in $U_{n}$ so that $U_{n}$ is multiply connected for all $n$. It is not difficult to see that $\left.f^{n}\right|_{U} \rightarrow \infty$ as $n \rightarrow \infty$. By (2), there exists a sequence $\left(r_{n}\right)$ tending to $\infty$ and a constant $C$ such that $\sigma_{n} \subset \operatorname{ann}\left(r_{n}, r_{n}^{C}\right)$, where $\operatorname{ann}(r, R)=\{z: r<|z|<R\}$ if $0 \leq r<R$. By Theorem 4 there exists a periodic point $z_{0}$ contained in $\operatorname{int}(\sigma)$, the interior of $\sigma$. It follows that for all $n$, some point of the periodic cycle to which $z_{0}$ belongs is contained in $\operatorname{int}\left(\sigma_{n}\right)$. Hence $D\left(0, r_{n}\right) \subset \operatorname{int}\left(\sigma_{n}\right)$ for sufficiently large $n$, where $D(a, r)=\{z:|z-a|<r\}$ for $r>0$ and $a \in \mathbb{C}$.

Suppose now that $U$ is preperiodic. Replacing $U$ by $U_{m}$ and $f$ by $f^{n}$ for suitable values of $m$ and $n$, we may assume without loss of generality that $U$ is invariant. We deduce from Lemma 7 that there exist a constant $A$ and a curve $\gamma$ tending to $\infty$ such that $|f(z)| \leq|z|^{A}$ for $z \in \gamma$. For sufficiently large $n, \sigma_{n}$ intersects $\gamma$; that is, we can find $w_{n} \in \sigma_{n} \cap \gamma$. Denoting by $M(r, f)$ the maximum modulus of $f$, that is, $M(r, f)=\max _{|z|=r}|f(z)|$, we deduce that

$M\left(r_{n}, f\right) \leq \max _{z \in \sigma_{n}}|f(z)| \leq r_{n+1}^{C} \leq\left(\min _{z \in \sigma_{n}}|f(z)|\right)^{C} \leq\left|f\left(w_{n}\right)\right|^{C} \leq\left|w_{n}\right|^{C A} \leq\left(r_{n}\right)^{C^{2} A}$.

This is a contradiction to the hypothesis that $f$ is transcendental and thus completes the proof of Theorem 9 .

The argument used above actually shows that certain classes of entire functions do not have multiply connected domains of normality at all. For example, using this method, one can obtain the following result.

Theorem 10. Suppose that $f \in E$ and that for all $\varepsilon>0$ there exists a curve $\gamma$ tending to $\infty$ such that $|f(z)| \leq M\left(|z|^{\varepsilon}, f\right)$ for $z \in \gamma$. Then all components of $F$ are simply connected. In particular, this is the case if $\log |f(z)|=O(\log |z|)$ as $z \rightarrow \infty$ through some path.

On the other hand, examples of entire functions with multiply connected components of the Fatou set are known. The first example was constructed in [7]; further examples can be found in [14, 17, 84].

Baker [17] gave an example of a transcendental entire function with an infinitely connected (and hence wandering) domain of normality. In the other examples cited, it is not clear what the connectivity of the multiply connected components is.

Question 7. Is there an entire transcendental function whose Fatou set has multiply connected components of finite connectivity?

4.5. Wandering domains. The first example of an entire function with a wandering domain was given by Baker [14]. We remark that the existence of wandering domains follows directly from his results in [7] (existence of multiply connected domains of normality) and [13] (multiply connected domains of normality are bounded), but [14] was written before [13] (although published later). 
Since then, many other examples have been constructed. The elementary examples

$$
f_{1}(z)=z-1+e^{-z}+2 \pi i
$$

and

$$
f_{2}(z)=z+\lambda \sin (2 \pi z)+1
$$

where $1+2 \pi \lambda=e^{2 \pi i \alpha}$ for suitable real numbers $\alpha$ have been given by Herman $([81$, p. $106 ; 130$, p. 414$]$; see also $[16$, pp. 564,567$])$. While the wandering domain in Baker's example [14] is multiply connected, $f_{1}$ and $f_{2}$ have simply connected wandering domains.

To prove that $f_{1}$ has a wandering domain, define $g(z)=z-1+e^{-z}$. (The function $g$ arises if we apply Newton's method to $h(z)=e^{z}-1$, that is, $g(z)=z-h(z) / h^{\prime}(z)$.) For $k \in \mathbb{Z}$ we define $z_{k}=2 \pi k i$. Then $z_{k}$ is a superattracting fixed point of $g$. Denote by $U_{k}$ the immediate attractive basin of $z_{k}$, that is, the component of $F(g)$ that contains $z_{k}$. One can show that $J(g)=J\left(f_{1}\right)$; compare [16, Lemma 4.5]. It follows that $U_{k}$ is also a component of $F\left(f_{1}\right)$, and we clearly have $f\left(U_{k}\right) \subset U_{k+1}$; that is, $U_{k}$ is wandering. It is not difficult to see that $U_{k}$ is simply connected for all $k$.

The proof that $f_{2}$ has a simply connected wandering domain is similar. Here $\alpha$ is chosen such that $z+\lambda \sin (2 \pi z)$ has a Siegel disc at zero. In this example, we obtain wandering domains $U_{k}$ containing $k \in \mathbb{Z}$. Here the $U_{k}$ have the additional feature that $\left.f\right|_{U_{k}}$ is univalent. Different examples of wandering domains with this property have been constructed by Eremenko and Lyubich [68, Example 2].

An example similar to $f_{1}$ and $f_{2}$ is given by

$$
f_{3}(z)=z+\lambda \sin z
$$

where $\lambda \in \mathbb{R}$ is chosen so that the forward orbit of each critical point consists only of critical points. For a discussion of this example, see [52, p. 222; 56, p. $290 ; 60$, p. 52]. Other examples of wandering domains with various additional properties have also been given. For example, Baker [17, Theorem 1] (see also [16, Theorem 5.2]) has shown that the order of an entire function with wandering domains may take any value.

In all examples mentioned so far, the iterates tend to $\infty$ in the wandering domain. It is well known (see [23, Lemma $2.1 ; 48$, p. $317 ; 71, \S 28]$ ) that there cannot exist nonconstant limit functions of $\left\{\left.f^{n}\right|_{U}\right\}$ if $U$ is a wandering domain of a meromorphic function $f$. Eremenko and Lyubich [68, Example 1] have constructed an entire function $f$ with a wandering domain $U$ such that the set of limit functions of $\left\{\left.f^{n}\right|_{U}\right\}$ contains an infinite number of finite constants. In this example, the constant limit functions have $\infty$ as a limit point; that is, $\infty$ is also a limit function of $\left\{\left.f^{n}\right|_{U}\right\}$. It is a well-known open problem [39, Problems 2.77 and 2.87] whether this is always the case.

Question 8. Let $U$ be a wandering domain of the transcendental meromorphic function $f$. Does there exist a sequence $\left(n_{k}\right)$ such that $\left.f^{n_{k}}\right|_{U} \rightarrow \infty$ as $k \rightarrow \infty$ ?

We remark that it has been shown in [33] that if $f \in E$ and $U$ is a wandering domain of $f$, then all finite limit functions of $\left\{\left.f^{n}\right|_{U}\right\}$ are contained in the derived set of $O^{+}\left(\operatorname{sing}\left(f^{-1}\right)\right)$. 
Finally, we mention that Baker, Kotus, and Lü $[22, \S 6]$ have modified the method of Eremenko and Lyubich to construct a function $f \in M$ which has a multiply connected wandering domain $U$ of preassigned connectivity such that the limit set of $\left\{\left.f^{n}\right|_{U}\right\}$ contains infinitely many finite constants.

4.6. Classes of functions without wandering domains. One of the most important results in the iteration theory of rational functions is the following theorem of Sullivan $[129,130]$.

Theorem 11. Rational functions do not have wandering domains.

Together with Theorems 6 and 7, this leads to a fairly complete description of the iterative behavior of rational functions on the Fatou set.

Sullivan's theorem has been extended to various classes of transcendental functions. We mention the following classes:

- $S=\{f: f$ has only finitely many critical and asymptotic values $\}$;

- $F=\left\{f: f\right.$ has a representation of the form $f(z)=z+r(z) e^{p(z)}$ where $r$ is rational and $p$ is a polynomial $\}$;

- $N=\left\{f: f\right.$ has finite order and $f^{\prime}(z)=r(z) e^{p(z)}(f(z)-z)$ where $r$ is rational and $p$ is a polynomial $\}$;

- $R=\left\{f: f^{\prime}(z)=r(z)(f(z)-z)^{2}\right.$ or $f^{\prime}(z)=r(z)(f(z)-z)(f(z)-\tau)$ where $r$ is rational and $\tau \in \mathbb{C}\}$.

The names of the different classes are somewhat arbitrary. According to Eremenko and Lyubich $[69$, p. 624] $S$ was chosen in honor of Speiser, who introduced this class in a different context. Class $N$ is of interest in connection with $N$ ewton's method (compare $\S 6$ ), and class $R$ consists of solutions of certain $R$ iccati equations (but is also of interest for Newton's method).

Theorem 12. Functions in $S, F, N$, and $R$ do not have wandering domains.

We note that all these classes contain the class of rational functions so that Theorem 12 may be considered as a generalization of Theorem 11 . The result that meromorphic functions in $S$ do not have wandering domains was proved by Baker, Kotus, and Lü [24]. This result had been obtained earlier by Eremenko and Lyubich [67, 70] and Goldberg and Keen [75] for $S \cap E$ and by Keen [91], Kotus [94], and Makienko [104] for $S \cap P$ (and, in fact, for the corresponding class of analytic self-maps of the punctured plane). For other subclasses of $S$, this had been proved by Baker [16, Theorem 6.2] and Devaney and Keen [59, p. 72].

The result that functions in $F$ do not have wandering domains was proved by Stallard [125]. The result for the classes $N$ and $R$ can be found in [31] and [35], respectively. In [35] the nonexistence of wandering domains is also proved for solutions of certain other differential equations.

The proofs in the papers cited above depend crucially on the fact that if $f$ is in one of the above classes, then there exist only finitely many singularities of $f^{-1}$ that are not contained in preperiodic components. (This is clear for $f \in S$ and easy to see for $f \in N$ and $f \in R$, but the proof for $f \in F$ is more involved; see [125].) It follows that if $U$ is a wandering domain, then there exists $n_{0}$ such that $U_{n} \cap \operatorname{sing}\left(f^{-1}\right)=\emptyset$ for $n \geq n_{0}$. Now two cases have to be distinguished: 
(i) $U_{n}$ is simply connected for all $n \geq n_{0}$.

(ii) $U_{m}$ is multiply connected for some $m \geq n_{0}$.

In case (i), one uses the ideas of Sullivan [130], who introduced quasiconformal mappings into the subject. We sketch the argument very briefly. Consider a quasiconformal homeomorphism of $U_{n_{0}}$ with complex dilatation $\mu$. Then $\mu$ can be extended to $\widehat{\mathbb{C}}$ in such a way that $\mu(f(z))=\mu(z) f^{\prime}(z) / \overline{f^{\prime}(z)}$ for all $z \in \mathbb{C}$. Then there exists a quasiconformal homeomorphism $\Phi$ of $\widehat{\mathbb{C}}$ that fixes 0,1 , and $\infty$ and whose complex dilatation is $\mu$. We now consider $f_{\Phi}=\Phi \circ f \circ \Phi^{-1}$ and observe that if $f$ is in one of the classes under consideration, then so is $f_{\Phi}$. This sharply limits the possibilities for $f_{\Phi}$, and-loosely speaking - a contradiction is obtained from the fact that there are many quasiconformal homeomorphisms of $U_{n_{0}}$ and hence many functions $\mu$ but not so many functions $f_{\Phi}$. For the details we refer to $[16,24,27]$.

In case (ii) it is not difficult to obtain a contradiction to Theorem 10 if $f$ is entire and contained in $S, F$, or $N$. A result similar to Theorem 10 can still be obtained if $f$ has finitely many poles, and this has been used to rule out case (ii) for meromorphic functions in $F$ and $N$; see [31] and [125] for details. For meromorphic functions in $S$, a different but still fairly elementary argument has been used; see [24]. The proof that case (ii) cannot occur for $f \in R$ is less elementary but uses results of Shishikura [122] obtained by quasiconformal surgery. We refer to [35] for the details.

Besides the classes contained in Theorem 12, there are some other classes of functions known to have no wandering domains. We mention that if $g$ is an analytic self-map of $\mathbb{C} \backslash\{0\}$, then there exist entire functions $f$ satisfying $\exp \circ f=g \circ \exp$. Thus results obtained for analytic self-maps of $\mathbb{C} \backslash\{0\}$ may be used to obtain results for entire functions $f$ that admit a representation of the above form. For example, one can prove using these ideas that if $p$ and $q$ are polynomials, then $f(z)=p\left(e^{z}\right)+q\left(e^{-z}\right)$ does not have wandering domains. For this and related results we refer to [16, 91, 94, 104].

If we combine the already mentioned result in [33] that all finite limit functions of $\left\{\left.f^{n}\right|_{U}\right\}$ are contained in the derived set of $O^{+}\left(\operatorname{sing}\left(f^{-1}\right)\right)$ if $f \in E$ and if $U$ is a wandering domain of $f$ with Theorem 15 in $\S 4.8$, we also obtain some classes of entire functions without wandering domains. We note that this is a fairly elementary way to obtain classes of functions without wandering domains, while the proofs of Theorems 11 and 12 use quasiconformal mappings.

Question 9. Is there a proof of Theorem 11 (and Theorem 12) that does not use quasiconformal mappings?

Some of the results concerning the nonexistence of wandering domains suggest that there are relations between wandering domains and singularities of the inverse function. In fact, similarly to $\S 4.3$ where we said that periodic components of the Fatou set (seem to) require one respectively two singularities, one may ask whether wandering domains require infinitely many of them, in a sense which still has to be made precise. More specifically, one may ask the following questions.

Question 10. Can a meromorphic function $f$ have wandering domains if all (or all but finitely many) points of $\operatorname{sing}\left(f^{-1}\right)$ are contained in preperiodic domains? 
Question 11. Let $f$ be a meromorphic function with a wandering domain $U$ such that $U_{n} \cap \operatorname{sing}\left(f^{-1}\right)=\emptyset$ for all $n \geq 0$. Is there some relation between $\partial U_{n}$ and $\operatorname{sing}\left(f^{-1}\right)$ ?

4.7. Baker domains. The first example of an entire function with a Baker domain was already given by Fatou [71, Example I], who considered the function

$$
f(z)=z+1+e^{-z}
$$

and proved that $f^{n}(z) \rightarrow \infty$ as $n \rightarrow \infty$ for $\operatorname{Re} z>0$, that is, the right halfplane is contained in an invariant Baker domain. An example of a Baker domain of higher period was given by Baker, Kotus, and Lü [23, p. 606], who showed that the function $f(z)=1 / z-e^{z}$ has a cycle $\left\{U_{0}, U_{1}\right\}$ of Baker domains such that $\left.f^{2 n}\right|_{U_{0}} \rightarrow \infty$ and $\left.f^{2 n}\right|_{U_{1}} \rightarrow 0$ as $n \rightarrow \infty$.

We list some general properties of Baker domains. Let $\left\{U_{0}, U_{1}, \ldots, U_{p-1}\right\}$ be a periodic cycle of Baker domains, and denote by $z_{j}$ the limit corresponding to $U_{j}$, that is, $f^{n p}(z) \rightarrow z_{j}$ for $z \in U_{j}$ as $n \rightarrow \infty$. Clearly, $f\left(z_{j}\right)=z_{j+1}$ if $z_{j} \neq \infty$. (Here, by definition, $z_{p}=z_{0}$.) It follows that there exists at least one $j \in\{0,1, \ldots, p-1\}$ such that $z_{j}=\infty$, and for all $j \in\{0,1, \ldots, p-1\}$ there exists $l=l(j) \in\{0,1, \ldots, p-1\}$ such that $f^{l}\left(z_{j}\right)=\infty$.

The $U_{j}$ contain curves $\gamma_{j}$ tending to $z_{j}$ such that $f^{p}\left(\gamma_{j}\right) \subset \gamma_{j}$ and $f^{p}(z) \rightarrow$ $z_{j}$ as $z \rightarrow z_{j}$ in $\gamma_{j}$. To see this, we proceed as in the proof of Lemma 7 and choose $w_{0} \in U_{0}$ and a curve $\sigma \subset U_{0}$ that joins $w_{0}$ and $f^{p}\left(w_{0}\right)$. We define $\gamma_{0}=\bigcup_{n=0}^{\infty} f^{n p}(\sigma)$ and $\gamma_{j}=f^{j}\left(\gamma_{0}\right)$ for $j \in\{1,2, \ldots, p-1\}$. Then the $\gamma_{j}$ have the desired properties. Moreover, $f(z) \rightarrow z_{j+1}$ as $z \rightarrow z_{j}$ in $\gamma_{j}$. We deduce that if $z_{j}=\infty$, then $z_{j+1}$ is an asymptotic value of $f$, the asymptotic path being contained in $U_{j}$.

We collect some of the above observations in the following theorem.

Theorem 13. Let $f$ be a meromorphic function, and let $\left\{U_{0}, U_{1}, \ldots, U_{p-1}\right\}$ be a periodic cycle of Baker domains of $f$. Denote by $z_{j}$ the limit corresponding to $U_{j}$, and define $z_{p}=z_{0}$. Then $z_{j} \in \bigcup_{n=0}^{p-1} f^{-n}(\infty)$ for all $j \in\{0,1, \ldots, p-1\}$, and $z_{j}=\infty$ for at least one $j \in\{0,1, \ldots, p-1\}$. If $z_{j}=\infty$, then $z_{j+1}$ is an asymptotic value of $f$.

Corollary 1. If $f$ has a cycle $\left\{U_{0}, U_{1}, \ldots, U_{p-1}\right\}$ of Baker domains such that $\left.f^{n}\right|_{U_{0}} \rightarrow \infty$, then $\infty$ is an asymptotic value of $f$. In particular, this is the case if $f$ has an invariant Baker domain.

Corollary 2. If $f$ has a cycle $\left\{U_{0}, U_{1}, \ldots, U_{p-1}\right\}$ of Baker domains such that $\left.f^{n}\right|_{U_{0}} \nrightarrow \infty$, then $f$ has a finite asymptotic value.

Corollary 1 can be found in $[59$, p. 75$]$ for maps with polynomial Schwarzian derivative.

Lemma 7 gives additional information about the asymptotic paths $\gamma_{j}$ and also answers the question how fast $f^{n p}(z)$ approaches $z_{j}$ for $z \in U_{j}$. In fact, if $U_{j}, z_{j}$, and $\gamma_{j}$ are as above, then $|z|^{1 / A} \leq\left|f^{p}(z)\right| \leq|z|^{A}$ for $z \in \gamma_{j}$ and $\log \log \left|f^{p n}(z)\right|=O(n)$ for $z \in D$ if $z_{j}=\infty$. If $U_{j}$ is simply connected and $z_{j}=\infty$, then we even have $|z| / A \leq\left|f^{p}(z)\right| \leq A|z|$ for $z \in \gamma_{j}$ and $\log \left|f^{p n}(z)\right|=O(n)$ for $z \in D$. Similar results may be obtained if $z_{j} \neq \infty$.

As already mentioned after Theorem 7, periodic cycles of Baker domains need not contain a singularity of $f^{-1}$. However, we have the following result. 
Theorem 14. If $f \in N$ or $f \in F$, then any periodic cycle of Baker domains contains a point of $\operatorname{sing}\left(f^{-1}\right)$.

This result was proved in [31] for $f \in N$, but the proof extends to the case that $f \in F$. The proof of Theorem 14 is fairly analogous to the proof that functions in $N$ (and $F$ ) do not have wandering domains. Therefore, it seems likely that the conclusion of Theorem 14 remains valid for functions in $R$. (This is certainly so for cycles of simply connected Baker domains, but in the multiply connected case some modification of the argument will have to be made.) For functions in $S$ we have a stronger result; see Corollary 4 in $\S 4.8$.

One way to prove that cycles of Leau domains contain a singularity of $f^{-1}$ is based on the solution of Abel's functional equation (cf. [108, §7]). Hinkkanen [83, Theorem 2] has shown that in certain cases this argument may also be used to prove that Baker domains contain singularities of $f^{-1}$.

4.8. Classes of functions without Baker domains. Eremenko and Lyubich [70] considered the class

$$
B=\left\{f: \operatorname{sing}\left(f^{-1}\right) \text { is bounded }\right\}
$$

and proved the following result.

Theorem 15. If $f \in E \cap B$, then there does not exist a component $U$ of $F(f)$ such that $\left.f^{n}\right|_{U} \rightarrow \infty$ as $n \rightarrow \infty$.

Corollary 3. If $f \in E \cap B$, then $f$ does not have Baker domains.

We note that the conclusion of Corollary 3 does not hold in general for $f \in M \cap B$. As an example, consider $f(z)=1 / z-e^{z}$. As already mentioned above, Baker, Kotus, and Lü [23, p. 606] proved that $f$ has a Baker domain of period 2 , and it is easy to check that $f \in M \cap B$. In this example, the critical values of $f$ accumulate at 0 , which is also one of the limits corresponding to the cycle of Baker domains.

The following result is a generalization of Corollary 3 to meromorphic functions.

Theorem 16. Let $f$ be a meromorphic function, and let $\left\{U_{0}, U_{1}, \ldots, U_{p-1}\right\}$ be a periodic cycle of Baker domains of $f$. Then $\infty$ is in the derived set of

$$
\bigcup_{j=0}^{p-1} f^{j}\left(\operatorname{sing}\left(f^{-1}\right)\right) \text {. }
$$

Corollary 4. Functions in $S$ do not have Baker domains.

Combining Corollary 4 with Theorem 12, we see that the iteration of functions in $S$ is in many ways analogous to that of rational functions and may thus be analyzed in a similar way.

For example, these results allow us to prove that the functions $\lambda z e^{z}, \lambda e^{z} / z$, and $\lambda \tan z$ satisfy $J=\widehat{\mathbb{C}}$ for certain values of $\lambda$, as mentioned in $\S 2.2$. In fact, all these functions are in $S$ and hence do not have wandering or Baker domains by Theorem 12 and Corollary 4 . For suitably chosen values of $\lambda$ we can achieve that the points of $\operatorname{sing}\left(f^{-1}\right)$ are either contained in $O^{-}(\infty)$, or they are preperiodic but not periodic. In view of Theorem 7 this implies that 
there are no immediate attractive basins, no Leau domains, no Siegel discs, and no Herman rings. Hence $J=\widehat{\mathbb{C}}$ for these $\lambda$. The above argument also shows that $J\left(e^{z}\right)=\widehat{\mathbb{C}}$.

For the proof of Theorem 16 we need the following lemma.

Lemma 8. Suppose $f \in B, p \geq 1$, and $0 \notin O^{-}(\infty)$. Then there exist a positive constant $R$ and a curve $\Gamma$ connecting 0 and $\infty$ such that $\left|f^{p}(z)\right| \leq R$ for $z \in \Gamma$.

We show first that if $r$ is sufficiently large, then there exists a curve $\Gamma$ connecting $\infty$ with some point in $\mathbb{C}$ such that $|f(z)|=r$ for $z \in \Gamma$. In fact, otherwise the components of $f^{-1}(D(0, r))$ are bounded for arbitrarily large $r$. Hence we can find $r_{1}$ and $r_{2}$ satisfying $0<r_{1}<r_{2}$ and $\operatorname{sing}\left(f^{-1}\right) \subset D\left(0, r_{1}\right)$ such that $f^{-1}\left(D\left(0, r_{2}\right)\right)$ has a bounded component which contains at least two components of $f^{-1}\left(D\left(0, r_{1}\right)\right)$. It follows that $f^{-1}\left(D\left(0, r_{2}\right)\right)$ contains a component of $f^{-1}\left(\operatorname{ann}\left(r_{1}, r_{2}\right)\right)$, which is at least triply connected. By the RiemannHurwitz formula (see, e.g., $[27, \S 5.4])$, this component contains a critical point of $f$; that is, $\operatorname{ann}\left(r_{1}, r_{2}\right)$ contains a critical value of $f$, contradicting the choice of $r_{1}$. Now we choose $r$ sufficiently large and a corresponding curve $\Gamma$ such that $\partial D(0, r) \cap O^{-}(\infty)=\emptyset$. Then there exists $R>0$ such that $\left|f^{p-1}(z)\right| \leq R$ for $|z|=r$. We deduce that $\left|f^{p}(z)\right| \leq R$ for $z \in \Gamma$. Increasing $R$ if necessary, we may assume that $\Gamma$ connects 0 and $\infty$. This completes the proof of Lemma 8.

To prove Theorem 16, we assume without loss of generality that $\left.f^{n p}\right|_{U_{0}} \rightarrow \infty$ as $n \rightarrow \infty$. Suppose that the conclusion of the theorem is false; that is, there exists a punctured neighborhood $N_{0}$ of $\infty$ which does not contain points of $\bigcup_{j=0}^{p-1} f^{j}\left(\operatorname{sing}\left(f^{-1}\right)\right)$. In particular, this implies that $f \in B$. We may assume without loss of generality that $0 \notin O^{-}(\infty)$ so that the hypotheses of Lemma 8 are satisfied. With $R$ and $\Gamma$ as in the conclusion of Lemma 8, we may suppose that $N_{0}=\{z:|z|>R\}$. In addition, we choose $R>|f(0)|$.

Suppose now that $w_{0} \in U_{0} \cap N_{0}$, and define $w_{1}=f^{p}\left(w_{0}\right)$. We may assume that $w_{1} \in U_{0} \cap N_{0}$, because otherwise we may replace $w_{0}$ by $f^{p n}\left(w_{0}\right)$ for a sufficiently large $n$. We introduce the abbreviation $g=f^{p}$. If $R$ has been chosen large enough, then the branch of $g^{-1}$ satisfying $g^{-1}\left(w_{1}\right)=w_{0}$ may be continued analytically in $N_{0}$. We define $u_{0}=\log w_{0}$ and $u_{1}=\log w_{1}$ for arbitrary branches of the logarithm. Then $\Phi=\log \circ g^{-1} \circ \exp$ may be defined as a single-valued function in the half-plane $H=\{z: \operatorname{Re} z>\log R\}$ such that $\Phi\left(u_{1}\right)=u_{0}$. Because $|g(z)| \leq R$ for $z \in \Gamma$, we have $\Phi(u) \notin \log \Gamma$ for all $u \in H$ and any branch of the logarithm. Hence $\Phi(H)$ does not contain a disc of radius greater than $\pi$ so that

$$
\left|\Phi^{\prime}(u)\right| \leq \frac{\pi}{B(\operatorname{Re} u-\log R)}
$$

where $B$ is Bloch's constant. (We do not need any estimate for $B$ here, just Bloch's theorem that $B>0$. Instead, we could also work with Landau's constant.) In terms of $g$, we find that

$$
\left|g^{\prime}(w)\right| \geq \frac{B|g(w)|(\log |g(w)|-\log R)}{\pi|w|} .
$$


Now we define $w_{n}=g^{n}\left(w_{0}\right)$ for $n \geq 2$, and we may assume that $w_{n} \in U_{0} \cap N_{0}$ for all $n$. (Otherwise, we may replace $w_{0}$ again by $g^{m}\left(w_{0}\right)$ for a sufficiently large $m$.) Then

$$
\left|g^{\prime}\left(w_{n}\right)\right| \geq c \frac{\left|g\left(w_{n}\right)\right| \log \left|g\left(w_{n}\right)\right|}{\left|w_{n}\right|}=c \frac{\left|w_{n+1}\right| \log \left|w_{n+1}\right|}{\left|w_{n}\right|}
$$

for some positive constant $c$ and all $n \geq 1$. Hence

$$
\left|\left(g^{n}\right)^{\prime}\left(w_{0}\right)\right|=\left|\prod_{j=0}^{n-1} g^{\prime}\left(w_{j}\right)\right| \geq \prod_{j=0}^{n-1} c \frac{\left|w_{j+1}\right| \log \left|w_{j+1}\right|}{\left|w_{j}\right|}=\frac{\left|w_{n}\right|}{\left|w_{0}\right|} \prod_{j=0}^{n-1} c \log \left|w_{j+1}\right|
$$

so that

$$
\frac{\left|\left(g^{n}\right)^{\prime}\left(w_{0}\right)\right|}{\left|w_{n}\right|} \rightarrow \infty
$$

as $n \rightarrow \infty$. We may assume that there exists a region $\Omega$ containing $w_{0}$ and $w_{1}$ such that $g^{n}(\bar{\Omega}) \subset U_{0} \cap N_{0}$ for all $n$. We apply Lemma 7 for $G=D=$ $\bigcup_{n=0}^{\infty} g^{n}(\Omega)$ and note that $\Gamma$ satisfies the hypotheses of this lemma. Hence $g^{n}(\Omega) \subset D\left(0, C\left|w_{n}\right|\right) \subset D\left(w_{n},(C+1)\left|w_{n}\right|\right)$ for some constant $C$ by (5). It follows that if we choose $r>0$ such that the disc around $w_{0}$ of radius $r$ is contained in $\Omega$, then

$$
\left|\left(g^{n}\right)^{\prime}\left(w_{0}\right)\right| \leq \frac{(C+1)\left|w_{n}\right|}{r}
$$

by Schwarz's lemma. This contradicts (9) and completes the proof of Theorem 16.

The above proof uses some of the ideas introduced by Eremenko and Lyubich [70] to prove Theorem 15. We sketch their proof of Theorem 15. First, we define again $N_{0}=\{z:|z|>R\}$. Using the methods of Lemma 8, one can show that the components of $f^{-1}\left(N_{0}\right)$ are simply connected and unbounded if $R$ is sufficiently large. This implies that if $D$ is a component of $f^{-1}\left(N_{0}\right)$ and if $A=\log D$ for some branch of the logarithm, then $\Psi=\log \circ f \circ \exp$ is a conformal map from $A$ onto $\Psi(A)$. We define $\Phi=\Psi^{-1}$ and find again that (8) holds. (Here we may replace Bloch's constant by Koebe's constant, which is equal to $\frac{1}{4}$, because $\Phi$ is univalent.) Suppose now that $w_{0} \in F$ and $f^{n}\left(w_{0}\right) \rightarrow \infty$. Define $u_{0}=\log w_{0}$. Similar to (9), we find that $\left|\left(\Psi^{n}\right)^{\prime}\left(u_{0}\right)\right| \rightarrow$ $\infty$. On the other hand, if $U$ is a sufficiently small neighborhood of $u_{0}$, then $\Psi^{n}(U)$ cannot contain a disc of radius larger than $\pi$. This is a contradiction to Koebe's (or Bloch's) theorem.

4.9. Completely invariant domains. Recall that a set $S$ is called completely invariant with respect to the meromorphic function $f$ if $O(S) \subset S$. One may ask in which cases a component of the Fatou set of a meromorphic function can be completely invariant. Such components are also called completely invariant domains.

It is classical that a rational function has at most two completely invariant domains [27, Theorem 5.6.1]. Here the number two is best possible, as shown by the simple example $f(z)=z^{2}$.

For transcendental entire functions, we have the following result of Baker [12]. 
Theorem 17. If $f \in E$, then $f$ has at most one completely invariant domain.

It is easy to find transcendental entire functions which have a completely invariant domain, for example, $f(z)=\lambda e^{z}$ has this property if $0<\lambda<1 / e$.

We mention the following question of Baker.

Question 12. Suppose $f \in E$ has a completely invariant domain $U$. Do we have $F(f)=U$ ?

Some results supporting the conjecture that the answer is "yes" can be found in [13, Theorem 2] and [70, §6]. In particular, it is shown in [70, Theorem 6] that this is the case if $f \in S \cap E$. We also note that Theorem 17 can be deduced from [13, Theorem 2; 70, Lemma 11].

Less is known about completely invariant domains of meromorphic functions. The example $f(z)=\tan z$ where $J=\mathbb{R} \cup\{\infty\}$ and where the upper and lower half-plane are completely invariant shows that there may be two completely invariant domains.

Question 13. Let $f$ be a meromorphic function. Can $f$ have more than two completely invariant domains?

A partial result was obtained by Baker, Kotus, and Lü [23, Theorem 4.5].

Theorem 18. If $f \in S$, then $f$ has at most two completely invariant domains.

If the answer to Question 13 is "no", one may also ask the following question.

Question 14. Suppose a meromorphic function $f$ has two completely invariant domains $U_{1}$ and $U_{2}$. Do we have $F(f)=U_{1} \cup U_{2}$ ?

\section{Properties of the Julia Set}

5.1. Cantor sets and real Julia sets. For rational functions the Julia set is often a Cantor set. (By definition a closed subset of $\widehat{\mathbb{C}}$ is called a Cantor set if it is perfect and totally disconnected.) For example, if $\lambda$ is not contained in the Mandelbrot set, then $J\left(z^{2}+\lambda\right)$ is a Cantor set.

For $f \in M$ it is also possible that $J(f)$ is a Cantor set. In fact, it was shown by Devaney and Keen [59, p. 62] that this is the case for $f(z)=\lambda \tan z$ if $-1<\lambda<1$ and $\lambda \neq 0$.

The following result, which is an immediate consequence of Theorem 9, says that this cannot happen for transcendental entire functions [13, p. 278, Corollary].

Theorem 19. If $f \in E$, then $J(f)$ contains nondegenerate continua.

For rational functions it is possible that the Julia set is a circle or a straight line; for example, $J\left(\frac{1}{2}(z-1 / z)\right)=\mathbb{R} \cup\{\infty\}$. This may also happen for $f \in M$. In fact, we have $J(\lambda \tan z)=\mathbb{R} \cup\{\infty\}$ if $\lambda \geq 1$; see [59, pp. 60-61]. For $f \in E$ this is impossible, as shown by the following result of Töpfer [133, §3].

Theorem 20. If $f \in E$, then $J(f)$ does not contain isolated Jordan arcs.

Here, by definition, a Jordan arc is called isolated (in $J$ ) if there exists an open set which contains the arc except for its endpoints but no other point of $J$. 
To prove Theorem 20, we suppose that such an arc exists and is parametrized by $\gamma:[0,1] \rightarrow \mathbb{C}$. By Theorem 4 , the repelling periodic points are dense in this arc. In view of Lemma 1 we may suppose that it contains a fixed point, say, $f\left(z_{1}\right)=z_{1}$ where $z_{1}=\gamma\left(t_{1}\right), t_{1} \in(0,1)$. We may also assume that $z_{1}$ is not exceptional so that $J=\overline{O^{-}\left(z_{1}\right)}$ by Lemma 4 . Hence there exist $t_{0}$ and $t_{2}$ satisfying $0<t_{0}<t_{1}<t_{2}<1$ and $n \geq 1$ such that $f^{n}\left(\gamma\left(t_{0}\right)\right)=f^{n}\left(\gamma\left(t_{2}\right)\right)=z_{1}$ and $f^{n}(\gamma(t)) \neq z_{1}$ for $t_{0}<t<t_{1}$ and $t_{1}<t<t_{2}$. We consider $C=$ $f^{n}\left(\gamma\left(\left[t_{0}, t_{2}\right]\right)\right)$. It follows from the assumption that $\gamma$ is isolated and from the complete invariance of $J$ that if $t \in\left(t_{0}, t_{2}\right)$, then there exists a neighborhood $N_{t}$ of $f^{n}(\gamma(t))$ such that $N_{t} \cap J \backslash C=\emptyset$. Because $f^{n}\left(\gamma\left(t_{0}\right)\right)=f^{n}\left(\gamma\left(t_{2}\right)\right)=z_{1}$, this is also true for $t=t_{0}$ and $t=t_{2}$. Hence $C$ has a neighborhood $N$ satisfying $N \cap J \backslash C=\emptyset$. This implies that $f(C) \subset C$, since $z_{1} \in C, J$ is completely invariant, and $C$ is connected. Hence $O^{+}(C) \subset C$. This is a contradiction, because $O^{+}(C) \backslash J$ contains at most the exceptional points of $f$ and is hence unbounded.

There are several other ways to see that $J(f)=\mathbb{R} \cup\{\infty\}$ is impossible for $f \in E$. One way is to observe that if this were the case, then the upper and lower half-plane were completely invariant with respect to $f^{2}$, contradicting Theorem 17. Another way to see that $J(f)=\mathbb{R} \cup\{\infty\}$ is impossible for $f \in E$ is to combine the complete invariance of $J(f)$ with a result of Edrei [65, p. 279] which says that if all roots of $f(z)=h_{n}$ are real for some unbounded sequence $\left(h_{n}\right)$ and an entire function $f$, then $f$ is a polynomial of degree at most 2 . More generally, one may use the above arguments to prove that the Julia set of an entire transcendental function cannot be contained in a finite set of straight lines; see [9].

We remark that Baker, Kotus, and Lü [21, Theorem 2], using a result of Čebotarev [43], have shown that if a transcendental meromorphic function $f$ satisfies $J(f)=\mathbb{R} \cup\{\infty\}$, then

$$
f(z)=\varepsilon\left(c z+d+\sum_{n=1}^{\infty} c_{n}\left(\frac{1}{a_{n}-z}-\frac{1}{a_{n}}\right)\right),
$$

where $c, d, c_{n}, a_{n} \in \mathbb{R}, \varepsilon= \pm 1, c \geq 0, c_{n}>0, a_{n} \neq 0$, and $\sum_{n=1}^{\infty} c_{n} / a_{n}^{2}<$ $\infty$. For further details concerning meromorphic functions satisfying $J \subset \mathbb{R} \cup$ $\{\infty\}$ we refer to [21].

\subsection{Points that tend to infinity. Eremenko [66] considered the set}

$$
I(f)=\left\{z: f^{n}(z) \rightarrow \infty \text { as } n \rightarrow \infty\right\} .
$$

If $f$ is a polynomial, then $I(f)$ is the immediate attractive basin of the superattracting fixed point $\infty$. In this case, we easily find that

$$
J(f)=\partial I(f) \text {. }
$$

Eremenko's main result in [66] is the following theorem.

Theorem 21. If $f \in E$, then $I(f) \neq \emptyset$.

Eremenko also shows that $I(f) \cap J(f) \neq \emptyset$. The proof of Theorem 21 is based on the Wiman-Valiron theory about the behavior of entire functions near points of maximum modulus; see for example [80, 135]. 
Once Theorem 21 is known, it is not difficult to prove that (10) holds for $f \in E$ as well. In particular, if an entire function $f$ does not have Baker domains (for example, if $f \in B \cap E$ ), then we have $J(f)=\overline{I(f)}$.

We mention two questions asked by Eremenko [66, pp. 343-344]. Suppose $f \in E$.

Question 15. Is every component of $I(f)$ unbounded?

Question 16. Can every point in $I(f)$ be joined with $\infty$ by a curve in $I(f)$ ?

Clearly, a positive answer to Question 16 would imply that the answer to Question 15 is also positive.

Eremenko [66, Theorem 3] proved that $\overline{I(f)}$ does not have bounded components, and he pointed out that a positive answer to Question 16 for a restricted class of functions follows from the results of Devaney and Tangermann [61].

5.3. Cantor bouquets. Devaney and Krych [60] have studied the Julia set of exponential functions. They find $\left[60\right.$, p. 50] that if $0<\lambda<1 / e$, then $J\left(\lambda e^{z}\right)$ is a so-called Cantor bouquet. We will define Cantor bouquets below but sketch the ideas only briefly and refer to $[50,52,53,56,61]$ for more details.

For a positive integer $N$ we consider the space $\Sigma_{N}$ of sequences of integers between $-N$ and $N$, that is,

$$
\Sigma_{N}=\left\{\left(s_{0}, s_{1}, s_{2}, \ldots\right): s_{j} \in \mathbb{Z},\left|s_{j}\right| \leq N\right\} .
$$

There is a natural topology that makes $\Sigma_{N}$ into a Cantor set. The shift $\sigma: \Sigma_{N} \rightarrow$ $\Sigma_{N}$ is defined by $\sigma\left(s_{0}, s_{1}, s_{2}, \ldots\right)=\left(s_{1}, s_{2}, s_{3}, \ldots\right)$. We call a closed subset $C_{N}$ of $\mathbb{C}$ a Cantor- $N$-bouquet of the meromorphic function $f$ if $f\left(C_{N}\right) \subset C_{N}$ and if there exists a homeomorphism $h: \Sigma_{N} \times[0, \infty) \rightarrow C_{N}$ with the following properties:

(i) $\left(\pi \circ h^{-1} \circ f \circ h\right)(s, t)=\sigma(s)$ for all $t \in[0, \infty)$, where $\pi$ : $\Sigma_{N} \times[0, \infty) \rightarrow \Sigma_{N}$ is the projection, that is, $\pi(s, t)=s$

(ii) $\lim _{t \rightarrow \infty} h(s, t)=\infty$;

(iii) $\lim _{n \rightarrow \infty} f^{n}(h(s, t))=\infty$ if $t>0$.

A Cantor- $N$-bouquet is similar to a Cantor set, but the components are curves tending to $\infty$ instead of points.

Given a sequence $C_{N}$ of Cantor- $N$-bouquets satisfying $C_{N} \subset C_{N+1}$, the set

$$
C_{\infty}=\overline{\bigcup_{N=1}^{\infty} C_{N}}
$$

is called a Cantor bouquet.

We indicate how a Cantor bouquet can be obtained for $E_{\lambda}(z)=\lambda e^{z}$ where $0<\lambda<1 / e$. Given $N \geq 1$, we choose $c>1$ such that $E_{\lambda}(c)>c+(2 N+1) \pi$ and consider the rectangles

$$
R_{j}=\{z: 1<\operatorname{Re} z<c,(2 j-1) \pi<\operatorname{Im} z<(2 j+1) \pi\}
$$

for $j \in\{-N,-N+1, \ldots, N\}$. For each $j$ we have

$$
E_{\lambda}\left(R_{j}\right)=\left\{z: \lambda e<|z|<\lambda e^{c},|\arg z|<\pi\right\} .
$$


Hence our choice of $c$ implies that $R_{k} \subset E_{\lambda}\left(R_{j}\right)$ if $j, k \in\{-N,-N+1$, $\ldots, N\}$. Define $R=\bigcup_{j=-N}^{N} R_{j}$ and

$$
\Lambda_{N}=\left\{z: E_{\lambda}^{N}(z) \in R \text { for all } n \geq 1\right\} .
$$

From the above observations we can deduce that $\Lambda_{N}$ is a Cantor set homeomorphic to $\Sigma_{N}$.

This construction yields the "endpoints" of the Cantor- $N$-bouquet, that is, the points in $h\left(\Sigma_{N} \times\{0\}\right)$. To obtain the curves attached to it, choose a point $w \in \Lambda_{N}$ and consider the set of all $z \in \mathbb{C}$ such that $E_{\lambda}^{n}(z)$ and $E_{\lambda}^{n}(w)$ lie in the same half-strip

$$
S_{j}=\{z: 1<\operatorname{Re} z<c,(2 j-1) \pi<\operatorname{Im} z<(2 j+1) \pi\}
$$

for all $n \geq 0$. This set then turns out to be a curve with the desired properties. We omit the details and refer to the papers cited above.

The method is not restricted to exponential functions. In fact, it is shown [61] that there exists a large class of functions, including, for example, $\sin z$ and $\cos z$, where the Julia set contains Cantor bouquets.

Besides the papers already mentioned we refer to $[1,41,106,120]$ for a further discussion of Cantor bouquets.

\section{NEWTON'S METHOD}

6.1. The unrelaxed Newton method. Let $g$ be a meromorphic function. Newton's method of finding the zeros of $g$ consists of iterating the meromorphic function $f$ defined by

$$
f(z)=z-\frac{g(z)}{g^{\prime}(z)} .
$$

In fact, if $\zeta$ is a zero of $g$, then $\zeta$ is an attracting fixed point of $f$, and vice versa. The simple zeros of $g$ correspond to the superattracting fixed points of $f$.

Clearly, if $z$ is close enough to $\zeta$, then $f^{n}(z)$ converges to $\zeta$ as $n \rightarrow \infty$. On the other hand, $f^{n}(z)$ cannot tend to a zero of $g$ if $z \in J(f)$, because $J(f)$ is completely invariant (under $f$ ). One may ask under which circumstances it is possible that $f^{n}(z)$ fails to converge to zeros of $g$ for some $z \in F(f)$ and, hence, for some open set of $z$-values. In view of Theorem 6 and because all fixed points of $f$ are attracting, this is possible only in one of the following cases:

(i) There exists $n \geq 0$ such that $f^{n}(z)$ is contained in a periodic cycle of immediate attractive basins, Leau domains, or Siegel discs. Here the minimal period of the cycle is greater than 1 .

(ii) There exists $n \geq 0$ such that $f^{n}(z)$ is contained in a periodic cycle of Herman rings.

(iii) There exists $n \geq 0$ such that $f^{n}(z)$ is contained in a periodic cycle of Baker domains.

(iv) $z$ is contained in a wandering domain.

We shall restrict here to the case that $g$ is entire and consider the case that $g$ is a polynomial first. Then $f$ is rational, and cases (iii) and (iv) do not occur. 
It follows from a result of Shishikura [122] that (ii) does not occur either. More precisely, Shishikura's result says that if a rational function has only one fixed point which is repelling or has multiplier 1 , then its Julia set is connected.

On the other hand, simple examples like $g(z)=z^{3}-z+1 / \sqrt{2}$ where 0 is a superattracting periodic point of minimal period 2 for $f$ show that case (i) can occur. From Theorem 7 and the fact that all finite fixed points of $f$ are attracting we can deduce that (i) cannot occur if $f^{n}(z)$ converges for all $z \in \operatorname{sing}\left(f^{-1}\right)$. (Theorem 7 also shows that (ii) does not occur if $f^{n}(z)$ converges for all $z \in \operatorname{sing}\left(f^{-1}\right)$.) Because $f^{\prime}(z)=g(z) g^{\prime \prime}(z) / g^{\prime}(z)^{2}$ and $\infty$ is a fixed point of $f$, we obtain the following result.

Theorem 22. Let $g$ be a polynomial, and let $f$ be defined by (11). Denote by $z_{1}, z_{2}, \ldots, z_{m}$ the zeros of $g^{\prime \prime}$ that are not zeros of $g^{\prime}$. If $f^{n}\left(z_{j}\right)$ converges for all $j \in\{1,2, \ldots, m\}$, then $f^{n}(z)$ converges to zeros of $g$ for all $z \in F(f)$.

The proof of Theorem 22 we have sketched above depends on Theorem 11. It is possible, however, to give a more elementary proof of Theorem 22. In fact, this result can be deduced from the work of Fatou [71, §30-31] and Julia [89, §5] (see also Smale [123, pp. 99-100]). As an example of where Theorem 22 applies, we mention real polynomials with only real zeros [26].

For a further discussion of Newton's method for polynomials we refer to $[77,97,132]$. If $g$ is transcendental, then so is $f$, except when $g=p e^{q}$ for polynomials $p$ and $q$. Newton's method for functions of this form has been studied in detail in [78].

We now consider the case that $g$ and $f$ are transcendental. Examples in [32] show that not only (i) can occur, but (iii) and (iv) can also occur. In fact, it was shown that case (iii) always occurs if $g$ tends to zero in some sector sufficiently fast, for example, if $g$ is of the form $g(z)=h(z) \exp \left(-z^{k}\right)$ for some positive integer $k$ and some entire function $h$ of order less than $k$ which does not have zeros in $|\arg z|<\varepsilon$ for some $\varepsilon>0$. An example where Newton's method leads to wandering domains is given by $g(z)=\exp \left(\frac{1}{2 \pi i} \int_{0}^{z} \cos ^{2}\left(e^{u}\right) d u\right)$; compare [32].

It is of interest to find classes of entire functions for which Newton's method behaves similarly to that for polynomials. In [31], Newton's method for functions $g$ of the form

$$
g(z)=\int_{0}^{z} p(t) e^{q(t)} d t+c
$$

where $p$ and $q$ are polynomials and where $c$ is a constant was studied. If $g$ has this form, then $f \in N$ and hence does not have wandering domains by Theorem 12. Also, every cycle of Baker domains contains a singularity of $f^{-1}$ by Theorem 14. Moreover, it was shown in [31] that if $g$ and $f$ are given by (12) and (11), then $f$ does not have finite asymptotic values. Hence we have the following result.

Theorem 23. If $g$ has the form (12) but is not of the form $g(z)=e^{a z+b}$ where $a$ and $b$ are constant, then the conclusion of Theorem 22 holds.

The case $g(z)=e^{a z+b}$ has to be excluded because then $f(z)=z-1 / a$, but we always assumed that $f$ is nonlinear. In fact, the conclusion of Theorem 23 is false in this case. 
Another class of entire functions where Newton's method does not lead to wandering domains are solutions of differential equations of the form $g^{\prime \prime}+$ $p g=0$ where $p$ is a polynomial. In this case, we have $f \in R$.

It seems likely that results of the type of Theorem 23 hold for a much wider class of functions. For instance, one may ask the following question which is related to Questions 5 and 10.

Question 17. Let $g$ be a meromorphic function, and let $f$ be defined by (11). Does the convergence of $f^{n}(z)$ for all $z \in \operatorname{sing}\left(f^{-1}\right)$ imply the convergence of $f^{n}(z)$ (to zeros of $g$ ) for all $z \in F(f)$ ?

6.2. The relaxed Newton method. As a generalization of Newton's method, one may consider the relaxed Newton method, which is given by iteration of

$$
f_{h}(z)=z-h \frac{g(z)}{g^{\prime}(z)}
$$

where $g$ is meromorphic and $h \in \mathbb{C},|h-1|<1$. Again, if $\zeta$ is a zero of $g$, then $\zeta$ is an attracting fixed point of $f$. For $h \neq 1$, however, $\zeta$ is not superattracting, but $f^{\prime}(\zeta)=1-h / m$ if $\zeta$ is a zero of $g$ of multiplicity $m$.

Clearly, the case $h=1$ corresponds to the unrelaxed Newton method considered in the previous section. Some of the results mentioned there extend to this more general case. For example, if $g$ is of the form (12) and if $f_{h}$ is defined by (13), then $f_{h}$ does not have wandering domains, and every cycle of Baker domains of $f_{h}$ contains a singularity of $f_{h}^{-1}$; see [31].

The relaxed Newton method may be viewed as a discretization of the differential equation

$$
\dot{z}=-\frac{g(z)}{g^{\prime}(z)} \text {. }
$$

This differential equation has been studied in a number of papers; compare the survey by Jongen, Jonker, and Twilt [88]. Similar to the immediate attractive basins with respect to the iteration of $f_{h}$, there are basins of attraction with respect to the differential equation attached to the zeros of $g$. For a zero $\zeta$ of $g$, we denote by $A^{*}(h, \zeta)$ the immediate basin of attraction of $\zeta$ with respect to the iteration of $f_{h}$, that is, the component of $F\left(f_{h}\right)$ that contains $\zeta$, and by $A(h, \zeta)$ the basin of attraction, that is, $A(h, \zeta)=\left\{z: \lim _{n \rightarrow \infty} f_{h}^{n}(z)=\zeta\right\}$. Clearly, $A^{*}(h, \zeta) \subset A(h, \zeta)$. By $B(\zeta)$ we denote the basin corresponding to the differential equation; that is, $B(\zeta)$ is the set of all $w \in \mathbb{C}$ such that there exists a solution $z:[a, b) \rightarrow \mathbb{C}$ of (14) satisfying $z(a)=w$ and $\lim _{t \rightarrow b} z(t)=\zeta$. Considering constant solutions of (14), we see that always $\zeta \in B(\zeta)$, provided we define $g(\zeta) / g^{\prime}(\zeta)=0$ for multiple zeros $\zeta$ of $g$.

We remark that if $g$ is rational, then

$$
\operatorname{meas}\left(\widehat{\mathbb{C}} \backslash \bigcup_{\{\zeta: g(\zeta)=0\}} B(\zeta)\right)=0,
$$

where meas $(\cdot)$ denotes Lebesgue measure on $\widehat{\mathbb{C}}$, while $\widehat{\mathbb{C}} \backslash \bigcup_{\{\zeta: g(\zeta)=0\}} A(h, \zeta)$ may contain open sets, as already pointed out in $\S 6.1$. It is of interest to study to what extent $A(h, \zeta)$ approximates $B(\zeta)$ if $h \rightarrow 0$. For rational functions 
$g$ and real values of $h$ this has been studied in detail in [76] and [107]. For example, it follows from the results obtained there that

$$
\lim _{h \rightarrow 0} \operatorname{meas}\left(\widehat{\mathbb{C}} \backslash \bigcup_{\{\zeta: g(\zeta)=0\}} A^{*}(h, \zeta)\right)=0 .
$$

We also mention [73], where it is proved that if $g$ is a polynomial, then

$$
\operatorname{meas}\left(\widehat{\mathbb{C}} \backslash \bigcup_{\{\zeta: g(\zeta)=0\}} A(h, \zeta)\right)=0
$$

for certain small (not necessarily real) values of $h$.

If $g$ is transcendental, then (15) and (16) need not be true. A simple example is provided by $g=p e^{q}$ if $p$ and $q$ are polynomials, $q$ nonconstant. More generally, it was shown in [32] that (15) and (16) do not hold if $g$ tends to zero in a suitable sector sufficiently fast. On the other hand, we have the following result proved in [32].

Theorem 24. Let $g$ be a meromorphic function. Suppose that $\operatorname{sing}\left(g^{-1}\right)$ is a discrete subset of $\mathbb{C}$ and that 0 is not an asymptotic value of $g$. Then (15) and (16) hold.

It seems likely that the conclusion of Theorem 24 remains valid for more general classes of functions.

Question 18. Is the hypothesis on the discreteness of $\operatorname{sing}\left(g^{-1}\right)$ necessary in Theorem 24?

\section{Miscellaneous topics}

In this paper, we have concentrated on describing some results in iteration theory that hold for all entire or meromorphic functions or at least for large classes of functions. Of course, it is also very important to consider specific examples. Already Fatou [72, pp. 358-369] studied the examples $f(z)=z+$ $1+e^{-z}$ and $f(z)=h \sin z+a$ (where $0<h<1$ and $a \in \mathbb{R}$ ) in detail, and Töpfer $[133, \S \S 5-6]$ described the Julia sets of the sine and cosine function.

A particularly important topic is to consider families of functions that depend on a parameter and to study how the iterative behavior varies as the parameter changes. In the iteration theory of rational functions, the family of quadratic polynomials and its bifurcation diagram, the Mandelbrot set, has been the object of much research. Among the transcendental functions, it is probably the exponential family $\left\{\lambda e^{z}: \lambda \in \mathbb{C} \backslash\{0\}\right\}$ that has received most attention. We have already mentioned some results in $\S 5.3$.

Define $E_{\lambda}(z)=\lambda e^{z}$. By Theorem 12 and Corollary $3, E_{\lambda}$ does not have wandering or Baker domains. Hence, in view of Theorem 7, the iterative behavior of $E_{\lambda}$ is largely determined by the forward orbit of 0 . In particular, $E_{\lambda}$ has at most one periodic cycle of immediate attractive basins, and if such a cycle exists, then it must contain 0 . If $E_{\lambda}^{n}(0) \rightarrow \infty$ or if the sequence $\left(E_{\lambda}^{n}(0)\right)_{n \geq 0}$ is preperiodic but not periodic, then $J\left(E_{\lambda}\right)=\widehat{\mathbb{C}}$. 
In $[25 ; 58 ; 70, \S 9]$ the iteration of $E_{\lambda}$ has been thoroughly investigated; for example, the sets

$$
D_{n}=\left\{\lambda: E_{\lambda} \text { has an attracting periodic cycle of minimal period } n\right\}
$$

have been studied in detail there. We omit these results here but just mention one open question.

Question 19. Is $\bigcup_{n=1}^{\infty} D_{n}$ a dense subset of $\mathbb{C}$ ?

This is an analogue to a well-known conjecture of Fatou $[71, \S 31$, p. 73] concerning rational functions. Some partial results concerning Question 19 can be found in $[50,51,137,140]$.

Of course, there are many other families that can be studied. For example, the functions

$$
\lambda \tan z, \frac{\lambda e^{z}}{e^{z}-e^{-z}}, \text { and } \frac{e^{z}}{\lambda e^{z}+e^{-z}}
$$

were studied in $[59, \S \S 2-4]$ for certain parameter values $\lambda$. We also mention $[64,85,86]$, where numerical studies concerning the iteration of transcendental meromorphic functions have been carried out.

There are many topics that have been left out. For example, we have not discussed ergodic problems. There are many papers on this topic for rational maps; see [69, Chapter 3] for a survey. Much less work has been done in this area for transcendental functions, but we mention [74, 101, 102, 115], which address these questions for the exponential function.

Another topic we have omitted is the investigation of the area and the Hausdorff dimension of Julia sets of transcendental functions. We refer to [70, $§ 7$; $103 ; 124 ; 126]$ for results in this direction.

\section{ACKNOWLEDGMENT}

I would like to thank Norbert Terglane for many useful discussions and valuable suggestions. I am also grateful to Alexander Eremenko, Aimo Hinkkanen, and Steffen Rohde for some helpful comments. Finally, I am indebted to two referees and to the editor, Richard Palais, for a number of helpful suggestions that led to an improvement of this paper.

\section{REFERENCES}

1. J. M. Aarts and L. Oversteegen, The geometry of Julia sets, Trans. Amer. Math. Soc. 338 (August 1993).

2. L. V. Ahlfors, Zur Theorie der Überlagerungsflächen, Acta Math. 65 (1935), 157-194.

3. __ Conformal invariants, McGraw-Hill, New York, 1973.

4. I. N. Baker, Fixpoints and iterates of entire functions, Math. Z. 71 (1959), 146-153.

5. (1959/60), 203-209.

6. The existence of fixpoints of entire functions, Math. Z. 73 (1960), 280-284.

7. Multiply connected domains of normality in iteration theory, Math. Z. 81 (1963), 206-214. 
8. (1964), 615-622.

9. 499-502.

10. _ Repulsive fixpoints of entire functions, Math. Z. 104 (1968), 252-256.

11. Limit functions and sets of non-normality in iteration theory, Ann. Acad. Sci. Fenn. Ser. A I Math. 467 (1970).

12. Completely invariant domains of entire functions, Mathematical Essays Dedicated to A. J. Macintyre, (H. Shankar, ed.), Ohio Univ. Press, Athens, Ohio, 1970, pp. 33-35.

13. $\longrightarrow$, The domains of normality of an entire function, Ann. Acad. Sci. Fenn. Ser. A I Math. 1 (1975), 277-283.

14. _ An entire function which has wandering domains, J. Austral. Math. Soc. Ser. A 22 (1976), 173-176.

15. The iteration of polynomials and transcendental entire functions, J. Austral. Math. Soc. Ser. A 30 (1981), 483-495.

16. Wandering domains in the iteration of entire functions, Proc. London Math. Soc. (3) 49 (1984), 563-576.

17. __ Some entire functions with multiply-connected wandering domains, Ergodic Theory Dynamical Systems 5 (1985), 163-169.

18. Iteration of entire functions: an introductory survey, Lectures on Complex Analysis, World Scientific, Singapore, New Jersey, London, and Hong Kong, 1987, pp. 1-17.

19. W Wandering domains for maps of the punctured plane, Ann. Acad. Sci. Fenn. Ser. A I Math. 12 (1987), 191-198.

20. - Infinite limits in the iteration of entire functions, Ergodic Theory Dynamical Systems 8 (1988), 503-507.

21. I. N. Baker, J. Kotus, and Y. Lü, Iterates of meromorphic functions. I, Ergodic Theory Dynamical Systems 11 (1991), 241-248.

22. __ Iterates of meromorphic functions II: Examples of wandering domains, J. London Math. Soc. (2) 42 (1990), 267-278.

23. —_ Iterates of meromorphic functions III: Preperiodic domains, Ergodic Theory Dynamical Systems 11 (1991), 603-618.

24. - Iterates of meromorphic functions IV: Critically finite functions, Results Math. 22 (1992), 651-656.

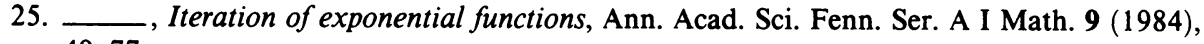
49-77.

26. B. Barna, Über die Divergenzpunkte des Newtonschen Verfahrens zur Bestimmung von Wurzeln von algebraischen Gleichungen II, Publ. Math. Debrecen 4 (1955/56), 384-397.

27. A. F. Beardon, Iteration of rational functions, Springer, New York, Berlin, and Heidelberg, 1991.

28. W. Bergweiler, On the number of fix-points of iterated entire functions, Arch. Math. 55 (1990), 558-563.

29. _ـ Periodic points of entire functions: proof of a conjecture of Baker, Complex Variables Theory Appl. 17 (1991), 57-72.

30. $\ldots$ On the existence of fixpoints of composite meromorphic functions, Proc. Amer. Math. Soc. 114 (1992), 879-880.

31. Newton's method and a class of meromorphic functions without wandering domains, Ergodic Theory Dynamical Systems (to appear).

32. W. Bergweiler, F. von Haeseler, H. Kriete, H.-G. Meier, and N. Terglane, Newton's method for meromorphic functions, Proceedings of the conference "Complex analysis and its applications", Hong Kong (to appear).

33. W. Bergweiler, M. Haruta, H. Kriete, H.-G. Meier, and N. Terglane, On the limit functions of iterates in wandering domains, Ann. Acad. Sci. Fenn. Ser. A I Math. (to appear). 
34. W. Bergweiler and S. Rohde, Omitted values in domains of normality, preprint.

35. W. Bergweiler and N. Terglane, Weakly repelling fixpoints and the connectivity of wandering domains, preprint.

36. P. Bhattacharyya, Iteration of analytic functions, Ph.D. thesis, Univ. of London, 1969.

37. P. Blanchard, Complex analytic dynamics on the Riemann sphere, Bull. Amer. Math. Soc. (N.S.) 11 (1984), 85-141.

38. L. E. Böttcher, Beiträge zu der Theorie der Iterationsrechnung, Inaugural dissertation, Leipzig, 1898.

39. D. A. Brannan and W. K. Hayman, Research problems in complex analysis, Bull. London Math. Soc. 21 (1989), 1-35.

40. H. Brolin, Invariant sets under iteration of rational functions, Ark. Mat. 6 (1967), 103-141.

41. W. D. Bula and L. Oversteegen, A characterization of smooth Cantor bouquets, Proc. Amer. Math. Soc. 108 (1990), 529-534.

42. L. Carleson and T. W. Gamelin, Complex dynamics, Springer, New York, Berlin, and Heidelberg, 1993.

43. N. G. Čebotarev, Über die Realität von Nullstellen ganzer transzendenter Funktionen, Math. Ann. 99 (1928), 660-686.

44. C.-T. Chuang, A simple proof of a theorem of Fatou on the iteration and fix-points of transcendental entire functions, Analytic Functions of One Complex Variable, (C.-C. Yang and C.-T. Chuang, eds.), Contemp. Math., vol. 48, Amer. Math. Soc., Providence, RI, 1985, pp. 65-69.

45. J. Clunie, The composition of entire and meromorphic functions, Mathematical Essays Dedicated to A. J. Macintyre (H. Shankar, ed.), Ohio Univ. Press, Athens, Ohio, 1970, pp. $75-92$.

46. H. Cremer, Über die Iteration rationaler Funktionen, Jahresber. Deutsche Math.-Ver. 33 (1925), 185-210.

47. — Zum Zentrumsproblem, Math. Ann. 98 (1926), 151-163.

48. __ Über die Schrödersche Funktionalgleichung und das Schwarzsche Eckenabbildungsproblem, Ber. Verh. Sächs. Akad. Wiss. Leipzig, Math.-Phys. Kl. 84 (1932), 291-324.

49. — Über die Häufigkeit der Nichtzentren, Math. Ann. 115 (1938), 573-580.

50. R. L. Devaney, Julia sets and bifurcation diagrams for exponential maps, Bull. Amer. Math. Soc. (N.S.) 11 (1984), 167-171.

51. _ Structural instability of $\exp (z)$, Proc. Amer. Math. Soc. 94 (1985), 545-548.

52. __ Dynamics of entire maps, Dynamical Systems and Ergodic Theory, Banach Center Publ., vol. 23, Polish Scientific Publishers, Warsaw, 1989, pp. 221-228.

53. $\longrightarrow$, An introduction to chaotic dynamical systems, second ed., Addison-Wesley, Redwood City, 1989.

54. _ Film and video as a research tool, Math. Intelligencer 11 (1989), 33-38.

55. _ Dynamics of simple maps, Chaos and Fractals. The Mathematics Behind the Computer Graphics (R. L. Devaney and L. Keen, eds.), Amer. Math. Soc., Providence, RI, 1989, pp. 1-24.

56. — $, e^{z}:$ Dynamics and bifurcations, Internat. J. Bifurcation Chaos 1 (1991), 287-308.

57. R. L. Devaney and M. B. Durkin, The exploding exponential and other chaotic bursts in complex dynamics, Amer. Math. Monthly 98 (1991), 217-232.

58. R. L. Devaney, L. R. Goldberg, and J. Hubbard, Dynamical approximation to the exponential map by polynomials, Preprint MSRI 10019-86, Mathematical Sciences Research Institute, Berkeley, CA, 1986.

59. R. L. Devaney and L. Keen, Dynamics of meromorphic maps with polynomial Schwarzian derivative, Ann. Sci. École Norm. Sup. (4) 22 (1989), 55-81.

60. R. L. Devaney and M. Krych, Dynamics of $\exp (z)$, Ergodic Theory Dynamical Systems 4 (1984), 35-52. 
61. R. L. Devaney and F. Tangermann, Dynamics of entire functions near the essential singularity, Ergodic Theory Dynamical Systems 6 (1986), 489-503.

62. A. Douady and J. H. Hubbard, Itération des polynômes quadratiques complexes, C. R. Acad. Sci. Paris Sér. I 294 (1982), 123-126.

63. É Étude dynamique des polynômes complexes. I \& II, Publ. Math. Orsay 84-02 (1984) \& 85-04 (1985).

64. N. Doual, J. L. Howland, and R. Vaillancourt, Selective solutions of transcendental equations, Computers Math. Appl. 22 (1991), 61-76.

65. A. Edrei, Meromorphic functions with three radially distributed values, Trans. Amer. Math. Soc. 78 (1955), 276-293.

66. A. E. Eremenko, On the iteration of entire functions, Dynamical Systems and Ergodic Theory, Banach Center Publ., vol. 23, Polish Scientific Publishers, Warsaw, 1989, pp. 339-345.

67. A. E. Eremenko and M. Yu. Lyubich, Iterates of entire functions, Soviet Math. Dokl. 30 (1984), 592-594; translation from Dokl. Akad. Nauk. SSSR 279 (1984).

68. __ Examples of entire functions with pathological dynamics, J. London Math. Soc. (2) 36 (1987), 458-468.

69. — The dynamics of analytic transforms, Leningrad Math. J. 1 (1990), 563-634.

70. _ Dynamical properties of some classes of entire functions, Ann. Inst. Fourier (Grenoble) 42 (1992), 989-1020.

71. P. Fatou, Sur les équations fonctionelles, Bull. Soc. Math. France 47 (1919), 161-271; 48 (1920), 33-94, 208-314.

72. __ Sur l'itération des fonctions transcendantes entières, Acta Math. 47 (1926), 337360.

73. M. Flexor and P. Sentenac, Algorithmes de Newton généralisés, C. R. Acad. Sci. Paris Sér. I Math. 308 (1989), 445-448.

74. E. Ghys, L. R. Goldberg, and D. P. Sullivan, On the measurable dynamics of $z \rightarrow e^{z}$, Ergodic Theory Dynamical Systems 5 (1985), 329-335.

75. L. R. Goldberg and L. Keen, $A$ finiteness theorem for a dynamical class of entire functions, Ergodic Theory Dynamical Systems 6 (1986), 183-192.

76. F. von Haeseler and H. Kriete, The relaxed Newton's method for polynomials, preprint.

77. F. von Haeseler and H.-O. Peitgen, Newton's method and complex dynamical systems, Acta Appl. Math. 13 (1988), 3-58; Newton's method and dynamical systems (H.-O. Peitgen, ed.), Kluwer Academic Publishers, Dordrecht, 1989, pp. 3-58.

78. M. Haruta, The dynamics of Newton's method on the exponential function in the complex domain, Ph.D. thesis, Boston Univ., 1992.

79. W. K. Hayman, Meromorphic functions, Clarendon Press, Oxford, 1964.

80. Math. Bull. (3) 17 (1974), 317-358.

81. M. Herman, Exemples de fractions rationelles ayant une orbite dense sur la sphère de Riemann, Bull. Soc. Math. France 112 (1984), 93-142.

82. $ـ$ Are there critical points on the boundary of singular domains?, Comm. Math. Phys. 99 (1985), 593-612.

83. A. Hinkkanen, Iteration and the zeros of the second derivative of a meromorphic function, Proc. London Math. Soc. (3) 65 (1992), 629-650.

84.

85. J. L. Howland and R. Vaillancourt, Attractive cycles in the iteration of meromorphic functions, Numer. Math. 46 (1985), 323-337.

86. J. L. Howland, A. Thompson, and R. Vaillancourt, On the dynamics of a meromorphic function, Appl. Math. Notes 15 (1990), 7-37. 
87. G. Jank and L. Volkmann, Einführung in die Theorie der ganzen und meromorphen Funktionen mit Anwendungen auf Differentialgleichungen, Birkhäuser, Basel, Boston, and Stuttgart, 1985.

88. H. Th. Jongen, P. Jonker, and F. Twilt, The continuous, desingularized Newton method for meromorphic functions, Acta Appl. Math. 13 (1988), 82-121; Newton's method and dynamical systems (H.-O. Peitgen, ed.), Kluwer Academic Publishers, Dordrecht 1989, pp. 82-121.

89. G. Julia, Sur l'itération des fonctions rationelles, J. Math. Pures Appl. (7) 4 (1918), 47-245.

90. _ـ Sur des problèmes concernant l'itération des fonctions rationelles, C. R. Acad. Sci. Paris Sér. I. Math. 166 (1918), 153-156.

91. L. Keen, Dynamics of holomorphic self-maps of $\mathbb{C}^{*}$, Holomorphic Functions and Moduli. I (D. Drasin, C. J. Earle, F. W. Gehring, I. Kra, and A. Marden, eds.), Springer, New York, Berlin, and Heidelberg, 1988.

92. _ـ Topology and growth of a special class of holomorphic self-maps of $\mathbb{C}^{*}$, Ergodic Theory Dynamical Systems 9 (1989), 321-328.

93. _ Julia sets, Chaos and Fractals. The Mathematics Behind the Computer Graphics (R. L. Devaney and L. Keen, eds.), Amer. Math. Soc., Providence, RI, 1989, pp. 57-74.

94. J. Kotus, Iterated holomorphic maps of the punctured plane, Dynamical Systems, (A. B. Kurzhanski and K. Sigmund, eds.), Lecture Notes Econom. and Math. Systems, vol. 287, Springer, Berlin, Heidelberg, and New York, 1987, pp. 10-29.

95. The domains of normality of holomorphic self-maps of $\mathbb{C}^{*}$, Ann. Acad. Sci. Fenn. Ser. A I Math. 15 (1990), 329-340.

96. B. Krauskopf, Convergence of Julia sets in the approximation of $\lambda e^{z}$ by $\lambda\left(1+\frac{z}{d}\right)^{d}$, Internat. J. Bifurcation Chaos (to appear).

97. H. Kriete, On the efficiency of relaxed Newton's method, Proceedings of the conference "Complex analysis and its applications", Hong Kong (to appear).

98. S. Lattès, Sur l'itération des substitutions rationelles et les fonctions de Poincaré, C. R. Acad. Sci. Paris Ser. I Math. 166 (1918), 26-28 (Errata: p. 88).

99. O. Lehto and K. I. Virtanen, Quasikonforme Abbildungen, Springer, Berlin, Heidelberg, and New York, 1965.

100. M. Yu. Lyubich, The dynamics of rational transforms: the topological picture, Russian Math. Surveys 41 (1986), 43-117; translation from Uspekhi Mat. Nauk. 41 (1986), 35-95.

101. __ Measurable dynamics of the exponential, Soviet Math. Dokl. 35 (1987), 223-226; translation from Dokl. Akad. Nauk. SSSR 292 (1987).

102. _ Measurable dynamics of the exponential, Sib. Math. J. 28 (1988), 780-793; translation from Sib. Mat. Zh. 28 (1987), 111-127.

103. C. McMullen, Area and Hausdorff dimension of Julia sets of entire functions, Trans. Amer. Math. Soc. 300 (1987), 329-342.

104. P. M. Makienko, Iterates of analytic functions of $\mathbb{C}^{*}$, Soviet Math. Dokl. 36 (1988), 418420; translation from Dokl. Akad. Nauk. SSSR 297 (1987).

105. R. M. May, Simple mathematical models with very complicated dynamics, Nature 261 (1976), 459-467.

106. J. C. Mayer, An explosion point for the set of endpoints of the Julia set of $\lambda \exp (z), \operatorname{Ergodic}$ Theory Dynamical Systems 10 (1990), 177-183.

107. H.-G. Meier, The relaxed Newton-iteration for rational functions: the limiting case, Complex Variables Theory Appl. 16 (1991), 239-260.

108. J. Milnor, Dynamics in one complex variable: introductory lectures, Stony Brook Institute for Mathematical Sciences, preprint 1990/5.

109. M. Misiurewicz, On iterates of $e^{z}$, Ergodic Theory and Dynamical Systems 1 (1981), 103-106.

110. P. Montel, Leçons sur les familles normales de fonctions analytiques et leurs applications, Gauthiers-Villars, Paris, 1927.

111. R. Nevanlinna, Analytic functions, Springer, Berlin, Heidelberg, and New York, 1970. 
112. H.-O. Peitgen and P. H. Richter, The beauty of fractals, Springer, Berlin, Heidelberg, New York, and Tokyo, 1986.

113. R. Pérez-Marco, Solution complète au problème de Siegel de linéarisation d'une application holomorphe au voisinage d'un point fixe (d'après J.-C. Yoccoz), Sém. Bourbaki 753 (1992).

114. H. Radström, On the iteration of analytic functions, Math. Scand. 1 (1953), 85-92.

115. M. Rees, The exponential map is not recurrent, Math. Z. 191 (1986), 593-598.

116. J. F. Ritt, On the iteration of rational functions, Trans. Amer. Math. Soc. 21 (1920), 348356.

117. P. C. Rosenbloom, L'itération des fonctions entières, C. R. Acad. Sci. Paris Sér. I Math. 227 (1948), 382-383.

118. D. Ruelle, Elements of differentiable dynamics and bifurcation theory, Academic Press, Boston, 1989.

119. C. L. Siegel, Iteration of analytic functions, Ann. of Math. (2) 43 (1942), 607-612.

120. M. Viana da Silva, The differentiability of the hairs of $\exp (z)$, Proc. Amer. Math. Soc. 103 (1988), 1179-1184.

121. M. Shishikura, On the quasi-conformal surgery of rational functions, Ann. Sci. École Norm. Sup. (4) 20 (1987), 1-29.

122. _ The connectivity of the Julia set and fixed point, preprint IHES/M/90/37, Institut des Hautes Études Scientifiques, 1990.

123. S. Smale, On the efficiency of algorithms of analysis, Bull. Amer. Math. Soc. (N.S.) 13 (1985), 87-121.

124. G. M. Stallard, Entire functions with Julia sets of zero measure, Math. Proc. Cambridge Philos. Soc. 108 (1990), 551-557.

125. _ A class of meromorphic functions with no wandering domains, Ann. Acad. Sci. Fenn. Ser. A I Math. 16 (1991), 211-226.

126. _ The Hausdorff dimension of Julia sets of entire functions, Ergodic Theory Dynamical Systems 11 (1991), 769-777.

127. N. Steinmetz, On Sullivan's classification of periodic stable domains, Complex Variables Theory Appl. 14 (1990), 211-214.

128. _ Rational iteration, Walter de Gruyter, Berlin, 1993.

129. D. Sullivan, Itération des fonctions analytiques complexes, C. R. Acad. Sci. Paris Sér. I Math. 294 (1982), 301-303.

130. _ Quasiconformal homeomorphisms and dynamics I. Solution of the Fatou-Julia problem on wandering domains, Ann. of Math. (2) 122 (1985), 401-418.

131. _ Quasiconformal homeomorphisms and dynamics III. Topological conjugacy classes of analytic endomorphisms, preprint IHES/M/83/1, Institut des Hautes Etudes Scientifiques, 1983.

132. S. Sutherland, Finding roots of complex polynomials with Newton's method, Ph.D. thesis, Boston Univ., 1989.

133. H. Töpfer, Über die Iteration der ganzen transzendenten Funktionen, insbesondere von $\sin z$ und $\cos z$, Math. Ann. 117 (1939), 65-84.

134. _ Komplexe Iterationsindizes ganzer und rationaler Funktionen, Math. Ann. 121 (1949), 191-222.

135. G. Valiron, Lectures on the general theory of integral functions, Édouard Privat, Toulouse, 1923.

136. C.-C. Yang and J.-H. Zheng, Further results on fixpoints and zeros of entire functions, Trans. Amer. Math. Soc. (to appear).

137. Zhuan Ye, Structural instability of exponential functions, preprint.

138. J.-C. Yoccoz, Théorème de Siegel, nombres de Brjuno et polynômes quadratiques, preprint, 1987. 
139. L_ Linéarisation des germes de difféomorphismes holomorphes de $(C, 0), \mathrm{C} . \mathrm{R}$. Acad. Sci. Paris Sér. I Math. 306 (1988), 55-58.

140. J. Zhou and Z. Li, Structural instability of the mapping $z \rightarrow \lambda \exp (z)\left(\lambda>e^{-1}\right)$, Sci. China Ser. A 30 (1989), 1153-1161.

Lehrstuhl II FÜr Mathematik, RWTH Aachen, D-52056 AACHEN, GeRmany

E-mail address: sf010be@dacth11.bitnet 